Research Article

\title{
Design of Attitude Control Systems for CubeSat-Class Nanosatellite
}

\author{
Junquan Li, Mark Post, Thomas Wright, and Regina Lee \\ Department of Earth \& Space Science and Engineering, York University, 4700 Keele Street, Toronto, ON, Canada M3J 1P3
}

Correspondence should be addressed to Junquan Li; junquanl@yorku.ca

Received 18 December 2012; Accepted 24 April 2013

Academic Editor: Sabri Cetinkunt

Copyright (C) 2013 Junquan Li et al. This is an open access article distributed under the Creative Commons Attribution License, which permits unrestricted use, distribution, and reproduction in any medium, provided the original work is properly cited.

\begin{abstract}
We present a satellite attitude control system design using low-cost hardware and software for a $1 \mathrm{U}$ CubeSat. The attitude control system architecture is a crucial subsystem for any satellite mission since precise pointing is often required to meet mission objectives. The accuracy and precision requirements are even more challenging for small satellites where limited volume, mass, and power are available for the attitude control system hardware. In this proposed embedded attitude control system design for a $1 \mathrm{U}$ CubeSat, pointing is obtained through a two-stage approach involving coarse and fine control modes. Fine control is achieved through the use of three reaction wheels or three magnetorquers and one reaction wheel along the pitch axis. Significant design work has been conducted to realize the proposed architecture. In this paper, we present an overview of the embedded attitude control system design; the verification results from numerical simulation studies to demonstrate the performance of a CubeSat-class nanosatellite; and a series of air-bearing verification tests on nanosatellite attitude control system hardware that compares the performance of the proposed nonlinear controller with a proportional-integral-derivative controller.
\end{abstract}

\section{Introduction}

The development of nanosatellites (with a mass of 1-10 kg) is currently a significant trend in the area of space science and engineering research. The development of CubeSat-class nanosatellites started in 1999 as a collaborative effort between California Polytechnic State University and Stanford University and has achieved great success as a way to efficiently construct and orbit small, inexpensive satellites using commercial technology. CubeSat, in general, is described as a class of nanosatellites ranging from $1 \mathrm{~kg}, 10 \times 10 \times 10 \mathrm{~cm}^{3}$, and upwards in $10 \mathrm{~cm}$ increments of length. Currently more than 50 research groups around the world are developing CubeSatclass nanosatellites for technology demonstration and scientific and student training missions. A solid model of a typical $1 \mathrm{U}$ CubeSat with attitude control systems (ACS) is shown in Figure 1.

Full-scale satellite attitude control systems are generally too large or too expensive to be installed in CubeSat-class nanosatellites [1], so passive attitude control systems have usually been used for nanosatellites in the past $[2,3]$. More active attitude control subsystems [4] in CubeSat-class nanosatellites have been implemented with the development of suitable actuators like magnetorquers (torque coils or torque rods) and small-sized reaction wheels $[5,6]$. An overview of the proposed ACS design adopted for this study is shown in Figure 2. Currently, commercial nanosatellite torque rods and reaction wheels are too expensive for use in many research nanosatellite projects. The contributions of this research are the development of ACS hardware from off-the-shelf components, complete simulation of the ACS system, and validation testing of the ACS system for attitude control in the lab environment.

In this paper, we first briefly describe the ACS hardware proposed for CubeSat-class nanosatellite missions. We outline the hardware development of the ACS actuators in Section 2. In particular, we describe the sizing and design of the magnetorquers. More discussions on the ACS hardware selection, design, and characterization for CubeSat-class nanosatellite missions, currently under development at York University, can be also found in [7-11]. In Sections 3 and 4, we describe the satellite system models and the results from the simulation study based on this design. In Section 5, we show the ground testing results of the hardware and software system. Section 6 includes future work that is planned. Section 7 concludes the paper. 


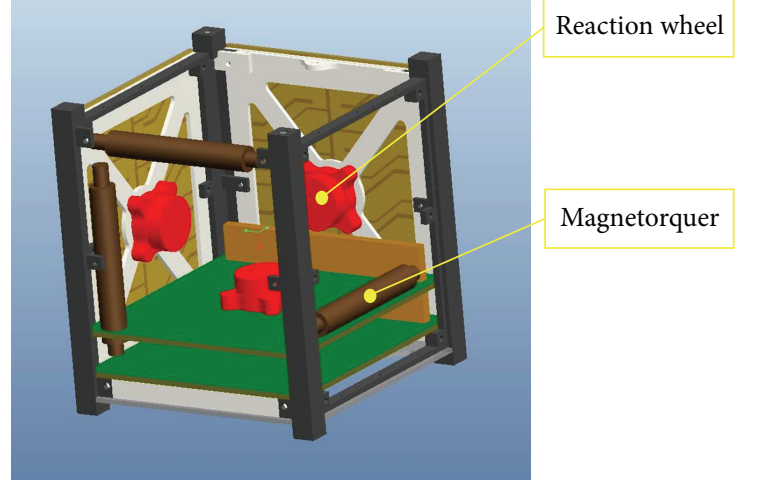

Figure 1: ACS (CubeSat-class nanosatellite with three reaction wheels and three torque rods).

\section{CubeSat ACS Hardware}

2.1. Attitude Sensors. Attitude magnetic sensor hardware in the current study consists of a Honeywell HMC5883L threeaxis MEMS magnetometer for magnetic field measurements. Angular rate information is obtained in three axes from three orthogonally mounted Analog Devices ADXRS614 MEMS gyroscopes. The attitude control system is managed by an AT91SAM9260 32-bit ARM9 microcontroller that runs embedded Linux, with $32 \mathrm{MB}$ SRAM, and $256 \mathrm{MB}$ NAND Flash attached for volatile and nonvolatile storage. All programming of control algorithms is accomplished in the $\mathrm{C}$ language using the GNU C compiler for the ARM processor. The system is designed for power-efficient operation because there is typically less than $3 \mathrm{~W}$ generated from the photovoltaics on a typical $1 \mathrm{U}$ CubeSat in low-earth orbit, and a battery must be used during eclipse periods.

2.2. Magnetorquer Design. Magnetic torque coils, also referred to as magnetorquers, in CubeSat-class nanosatellites provide baseline control in many small satellites. They are commercially available in two typical configurations: in loose coils of flat-wound wire and in tightly wound coils around a permalloy rod. The rod configuration is often preferred because of its compactness and rigidity and the use of highpermeability, $\mu$, materials for the core. To meet the mass and power requirements of a nanosatellite, a maximum mass, $M$, of $30 \mathrm{~g}$ and a conservative maximum power draw, $P$, of $0.2 \mathrm{~W}$ were set, and a typical maximum supply voltage, $V$, between $3.7 \mathrm{~V}$ and $4.2 \mathrm{~V}$ was assumed to avoid having to step up voltage on power components. After combining the power and mass equations (1), where $R$ is resistance, $W_{w}$ is the resistivity of the wire, and $\rho$ is the density, and solving for the core radius, $r_{c}$, and length, $l_{c}$,

$$
\begin{gathered}
R=\frac{V^{2}}{P}, \\
l_{w}=\frac{R}{W_{w}}, \\
M=\pi r_{w}^{2} l_{w} \rho_{w}+\pi r_{c}^{2} l_{c} \rho_{c} .
\end{gathered}
$$

The power and mass constraints were applied using (1), and a power value was estimated as the lesser of $0.2 \mathrm{~W}$ and the power dissipation was achieved at the maximum current for the wire

$$
\begin{gathered}
r_{c}^{2} l_{c}=\frac{M}{\pi \rho_{c}}-\frac{\rho_{w}}{\rho_{c}} \frac{r_{w} V^{2}}{P W_{w}}, \\
N_{d}=\frac{4\left[\ln \left(l_{c} / r_{c}\right)-1\right]}{\left(l_{c} / r_{c}\right)^{2}-4 \ln \left(l_{c} / r_{c}\right)}, \\
D=\frac{r_{c} V}{2 W_{w}}\left[1+\frac{\mu_{r}-1}{1+\left(\mu_{r}-1\right) N_{d}}\right] .
\end{gathered}
$$

Using (2) from the well-known solenoid equation and the relations derived in [12], where $N_{d}$ is the demagnetizing factor, a parametric analysis of the effect of core length and core radius on magnetic dipole moment, $D$, was used to determine the optimal length and radius of the core material, given the wire thickness and corresponding length to satisfy the power and dipole moment requirements. Figure 3 illustrates the effect of core sizing on generated magnetic moment. The number of turns is implicitly determined, as the dipole moment of the torque rod is independent of the number of turns of wire, and the core radius is also constrained to sizes that are commercially available.

To maximize the field generated with the dimensions while satisfying the design constraints, a prototype magnetorquer was designed with $70 \mathrm{~mm}$ long permalloy core and 36 AWG wire to provide a maximum load power of $200 \mathrm{~mW}$ at $4.2 \mathrm{~V}$. The design parameters of the constructed torque rods are shown in Table 1.

In order to precisely wind 36 AWG wires around a core, a coil winding machine was designed and assembled using stepper motors and L298 H-bridge drivers controlled by an ATMega644P microcontroller. A permalloy core is rotated by one motor, while another positions a plastic feeder guide from the wire spool. The winder, shown with a completed torque rod in Figure 4, allows a torque rod to be automatically wound by setting the number of turns required, length of the core, and thickness of the wire, which determines the ratio of winding speed to feeder speed.

2.3. Reaction Wheel Design. Pointing and slew maneuvering of satellites are often accomplished by a motorized rotating mass such as a reaction wheel and momentum wheel, which provide maneuvering torque and momentum storage [8]. Reaction wheels can provide a high degree of attitude control accuracy with the limitation that the wheel may reach saturation after continued use, requiring an additional momentum control method such as magnetorquers to desaturate the wheel in a process known as momentum dumping.

Each of the reaction wheels in the proposed ACS system consists of a steel cylinder that is press-fitted to the shaft of a Faulhaber brushless flat micromotor. Design choices for the motor were limited to inexpensive commercial motors with low-power consumption, and the reaction wheels were sized to provide maximum momentum storage given the mass and volume constraints of a $1 \mathrm{U}$ CubeSat. Three reaction wheels 


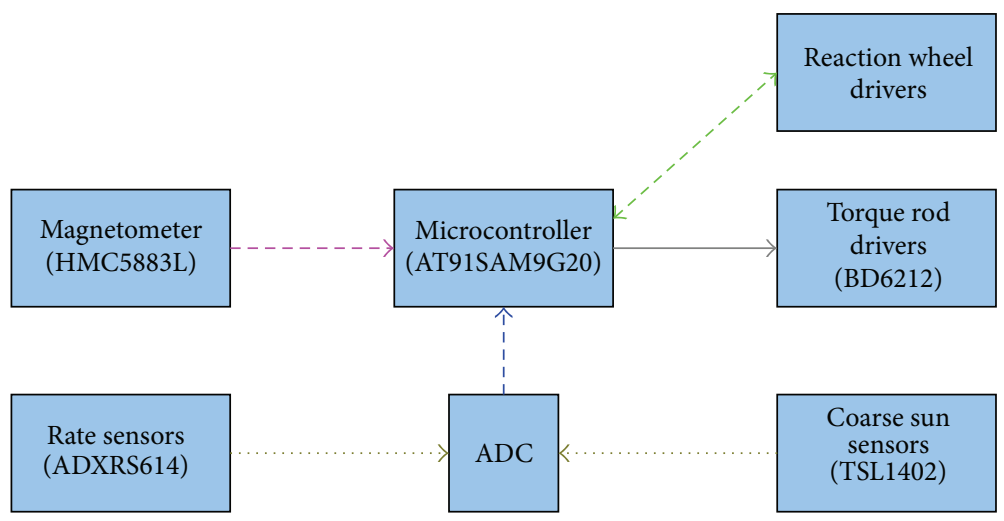

FIGURE 2: Overview of proposed ACS design for a CubeSat-class nanosatellite.

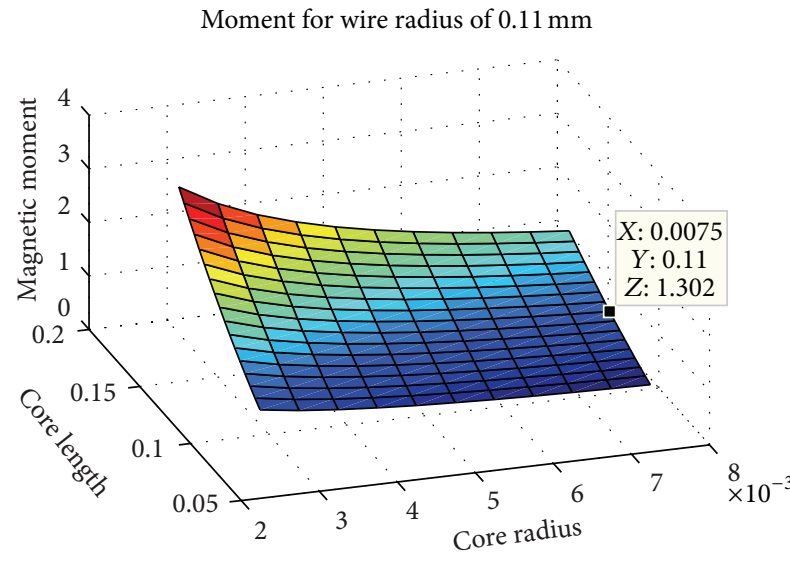

FIgURE 3: Magnetorquer sizing surface.

TABLE 1: Magnetorquer parameters.

\begin{tabular}{lcc}
\hline Parameter & Value & Unit \\
\hline Maximum dipole moment & 0.37 & $\mathrm{~A} \mathrm{~m}^{2}$ \\
Total mass & 28 & $\mathrm{~g}$ \\
Number of turns & 6063 & \\
Core diameter & 5.7 & $\mathrm{~mm}$ \\
Wire diameter & 0.127 & $\mathrm{~mm}$ \\
Wire resistance & 121 & $\Omega$ \\
Maximum current & 34.7 & $\mathrm{~mA}$ \\
\hline
\end{tabular}

can be used in the ACS if maximum control authority is required. Table 2 shows the design parameters of the reaction wheels used on the proposed ACS [13]. A completed reaction wheel assembly is shown in Figure 5.

2.4. Electronic Integration of ACS Components. To control the reaction wheel and magnetorquers hardware and house the attitude sensors and actuator drivers, a printed circuit board (PCB) was fabricated, shown in Figure 6. The board stacks with existing PC/104 sized on-board computer (OBC) hardware and provides both $\mathrm{I} / \mathrm{O}$ breakout and power supplies for the ACS hardware. It contains $3.3 \mathrm{~V}$ and $5 \mathrm{~V}$ switching supplies for the ACS sensors, as well as external interfaces for a battery and radio to be used specifically for air-bearing
TABLE 2: Reaction wheel parameters.

\begin{tabular}{lcc}
\hline Parameter & Value & Unit \\
\hline Rotor mass & 0.214 & $\mathrm{~kg}$ \\
Moment of inertia (axial) & $9.41 \times 10^{-5}$ & $\mathrm{Kg} \mathrm{m}^{2}$ \\
Moment of inertia (transverse) & $5.02 \times 10^{-5}$ & $\mathrm{~kg} \mathrm{~m}^{2}$ \\
Motor shaft Torque & $6.0 \times 10^{-4}$ & $\mathrm{~N} \mathrm{~m}$ \\
Maximum speed & 1539 & $\mathrm{rad} / \mathrm{s}$ \\
Supply voltage & $3.7-4.2$ & $\mathrm{~V}$ \\
\hline
\end{tabular}

ACS testing. The board makes a HMC5883 three-axis magnetometer, an ADXL345 3-axis accelerometer, and an ITG32003 -axis MEMS rate gyroscope available on the OBC $\mathrm{I}^{2} \mathrm{C}$ bus. Primary rate sensing for attitude control is accomplished by three independent ADXRS614 rate gyro units oriented on orthogonal axes by means of right-angle IC sockets and connected to the first three ADC channels on the OBC. This allows accurate high-speed sampling of rotation rates for use by the attitude controller. To drive the magnetorquers, three BD6212 integrated H-bridges are used, controlled by three PWM channels from the OBC and three general purpose IO pins for current direction control. To allow one PWM signal and one direction pin to control each $\mathrm{H}$-bridge, the inputs are demultiplexed by a SN74LVC1G8 tristate output demultiplexer and pull-up resistors. In full operation, the board draws up to $100 \mathrm{~mW}$ of power on average, though components can be shut down as needed to conserve power if not in use.

\section{System Models}

3.1. Attitude Equations of Motions. In this section, the satellite is modelled as a rigid body with actuators that provide torques about three mutually perpendicular axes that defines a body-fixed frame. The equations of motion $[14,15]$ are given by

$$
\widehat{J} \dot{\omega}_{b}=-\omega^{\times}\left(J_{S} \omega_{b}+A_{i} J_{w} \Omega\right)+A_{i} \tau_{c}+\tau_{m}+\tau_{d},
$$

where $\omega_{b}=\left(\omega_{b 1} \omega_{b 2} \omega_{b 3}\right)^{T}$ is the angular velocity of the satellite expressed in the body frame. $J_{s}$ is the inertia matrix of the satellite. $J_{w}$ is the inertia matrix of the reaction wheel and $\widehat{J}=J_{s}-A_{i} J_{w} A_{i}^{T} . A_{i}$ is the layout matrix of the reaction 

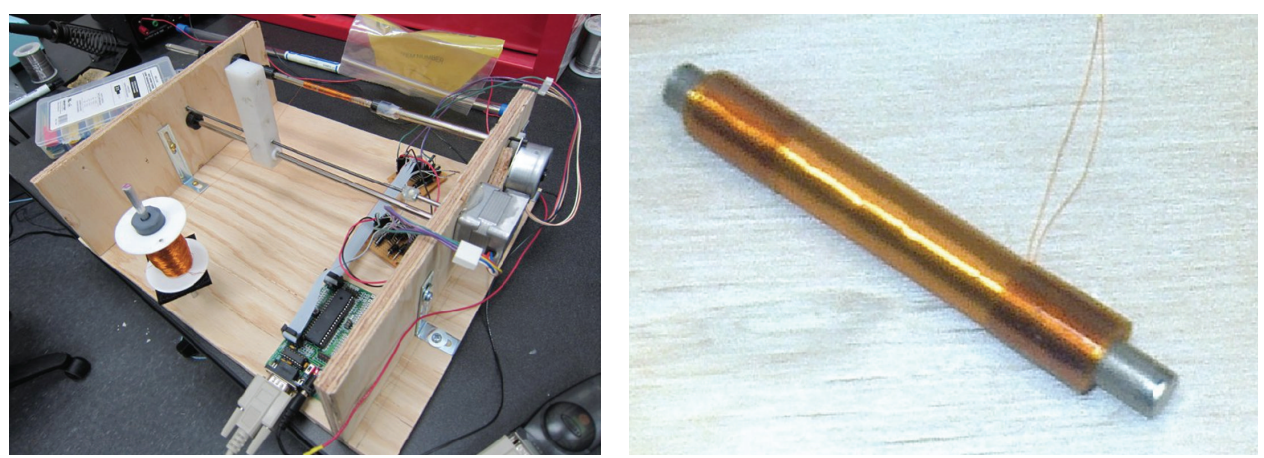

FIGURE 4: Magnetorquer winding apparatus and completed torque rod.

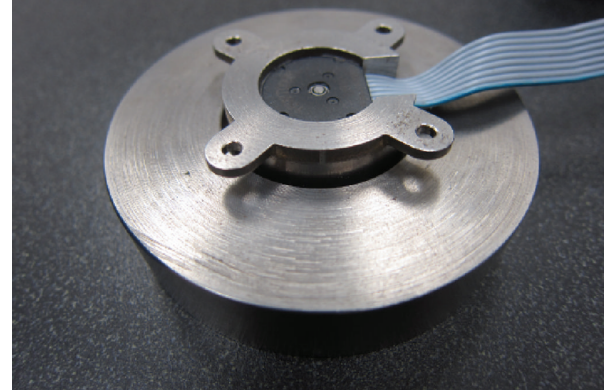

FIGURE 5: Reaction wheel assembly.

wheels whose columns represent the influence of each wheel on the angular acceleration of the satellite. $\Omega$ is the velocity of a reaction wheel, $\tau_{c}$ is the torque control provided by the reaction wheel, $\tau_{m}$ is the torque control provided by the magnetorquers, and $\tau_{d}$ is the bounded external disturbance, which is a sum of the gravity gradient $\tau_{\text {gravity }}$, aerodynamic $\tau_{\text {aero }}$, and solar radiation pressure $\tau_{\text {solar }}$ disturbances.

The gravity gradient disturbance is $\tau_{\text {gravity }}=$ $3{\sqrt{\mu_{e} / a^{3}}}^{2} C J_{s} C$, where $\mu$ is the gravitational parameter of the Earth, $a$ is the semimajor axis of the orbit, and $C_{k}$ is the direction cosine matrix in terms of quaternions.

The aerodynamic disturbance is $\tau_{\text {aero }}=C_{d} / 2 \rho v^{2} A L$, where $C_{d}$ is the coefficient of drag for a flat plate, $A$ is the cross-sectional area causing aerodynamic drag, $v$ is the satellite velocity, $L$ is the distance between the centre of pressure and the centre of gravity, and $\rho$ is the atmospheric density related to the altitude.

The solar radiation pressure disturbance is $\tau_{\text {solar }}=$ $F_{s} / c A_{s}(1+r) L$, where $F_{s}$ is the solar constant at the Earth's orbital distance from the Sun, $c$ is the speed of light, $A_{s}$ is the illuminated surface area, and $r$ is the surface reflectance.

3.2. Attitude Kinematics. The satellite attitude kinematics is represented using quaternions

$$
\dot{q}=\frac{1}{2}\left(\begin{array}{c}
q_{4} I_{3 \times 3}+\bar{q}^{\times} \\
-\bar{q}^{T}
\end{array}\right) \omega_{b} \equiv \frac{1}{2} A(q) \omega_{b},
$$

where $q=\left(\bar{q}^{T}, q_{4}\right)^{T}=\left(q_{1}, q_{2}, q_{3}, q_{4}\right)^{T}$.

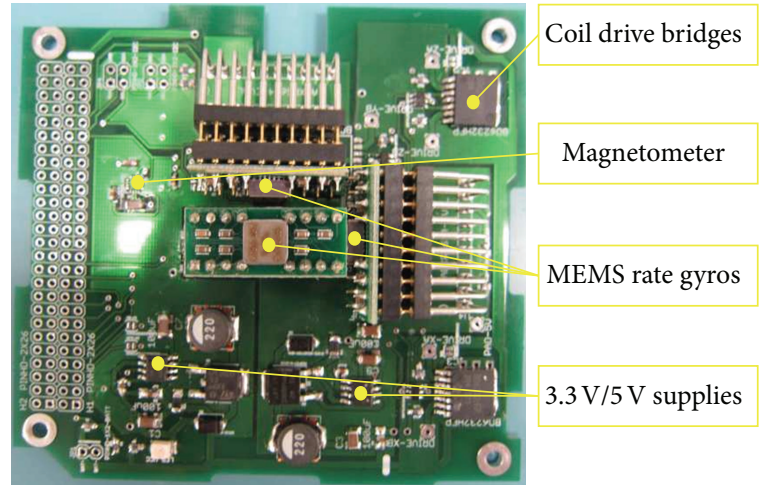

FIGURE 6: ACS sensor and actuator board.

In terms of Euler angles, we can also express the satellite attitude as

$$
\left[\begin{array}{c}
\dot{\psi} \\
\dot{\alpha} \\
\dot{\gamma}
\end{array}\right]=\left[\begin{array}{ccc}
1 & \sin (\psi) \tan (\alpha) & \cos (\psi) \tan (\alpha) \\
0 & \cos (\psi) & -\sin (\psi) \\
0 & \sin \left(\frac{\psi}{\cos (\alpha)}\right) & \frac{\cos (\psi)}{\cos (\alpha)}
\end{array}\right] \omega_{b},
$$

where $\psi$ is the roll angle about the $x$-axis, $\alpha$ is the pitch angle about the $y$-axis, and $\gamma$ is the yaw about the $z$-axis.

3.3. Sensor Models. Magnetic field vectors are obtained in the orbit reference frame

$$
\begin{aligned}
B_{1}= & \frac{M_{e}}{r_{0}^{3}} \\
& \times\left[\cos \left(\omega_{0} t\right)\left(\cos (\epsilon) \sin (i)-\sin (\epsilon) \cos (i) \cos \left(\omega_{e} t\right)\right)\right. \\
& \left.\quad-\sin \left(\omega_{0} t\right) \sin (\epsilon) \sin \left(\omega_{e} t\right)\right], \\
B_{2}= & \frac{-M_{e}}{r_{0}^{3}}\left[\left(\cos (\epsilon) \cos (i)+\sin (\epsilon) \sin (i) \cos \left(\omega_{e} t\right)\right)\right], \\
B_{3}= & \frac{3 M_{e}}{r_{0}^{3}} \\
& \times\left[\sin \left(\omega_{0} t\right)\left(\cos (\epsilon) \sin (i)-\sin (\epsilon) \cos (i) \cos \left(\omega_{e} t\right)\right)\right. \\
& \left.\quad-2 \sin \left(\omega_{0} t\right) \sin (\epsilon) \sin \left(\omega_{e} t\right)\right],
\end{aligned}
$$


where $\omega_{0}$ is the angular velocity of the orbit with respect to the inertial frame, $r_{0}$ is the distance from the center of the satellite to the center of the Earth, $i$ is the orbit inclination, $\varepsilon$ is the magnetic dipole tilt, $\omega_{e}$ is the spin rate of the Earth, and $M_{e}$ is the magnetic dipole moment of the Earth. The magnetometer model is

$$
H=C_{k}\left[\begin{array}{l}
B_{1} \\
B_{2} \\
B_{3}
\end{array}\right]+\eta_{m}+b_{m},
$$

where $C_{k}$ is the direction cosine matrix in terms of quaternions, $\eta_{m}$ is the zero mean Gaussian white noise of the magnetometer, $B$ is the vector formed with the components of the Earth's magnetic field in the body frame of the reference, and $B=C_{k}\left[\begin{array}{l}B_{1} \\ B_{2} \\ B_{3}\end{array}\right] \cdot b_{m}$ is the magnetometer bias.

The angular velocity is measured from three rate gyroscopes. The model is given by

$$
\begin{aligned}
& \omega_{g}=\omega+b_{g}+\eta_{g}, \\
& \dot{b}_{g}=-k_{f} b_{g}+\eta_{f},
\end{aligned}
$$

where $\omega_{g}$ is the output of a gyroscope and $\omega$ is the real angular rate of the gyro. $\eta_{g}$ and $\eta_{f}$ are Gaussian white noise. $b_{g}$ is the random drift of the gyro and $k_{f}$ is the drift constant.

3.4. Actuator Models. Reaction wheels are widely used to perform precise satellite attitude maneuvers because they allow continuous and smooth control of internal torques. Torques are produced on the satellite by accelerating or decelerating the reaction wheels. Let the torque demanded by the satellite be denoted as $\tau_{c}$, where $\tau_{c}=J_{w}\left(\dot{\Omega}+A_{i} \dot{\omega} b\right)$. The input voltage $e_{a}$ required to control the actuator dynamics of the reaction wheel can be written as

$$
e_{a}=k_{b} \Omega-R_{b} k_{t}^{-1}\left(A_{i}^{\prime} \tau_{c}\right),
$$

where $k_{t}$ is the motor torque constant, $k_{b}$ is the back-EMF constant, $R_{b}$ is the armature resistance, and friction in the reaction wheels is ignored. The maximum voltage of the reaction wheel is $4.2 \mathrm{~V}$, and a dead zone for the reaction wheel is estimated to be below $1 \mathrm{~V} . k_{t}$ is $0.0082, k_{b}$ is $0.007, R_{b}$ is 0.5 , and the moment of inertia of the reaction wheel is $0.0001 \mathrm{~kg} \mathrm{~m}^{2}$.

\section{Control Law Design and Simulation Results}

4.1. Satellite Attitude Control Laws. Magnetic control has been used over many years $[16,17]$ for small spacecraft attitude control. The main drawback of magnetic control is that magnetic torque is two-dimensional and it is only present in the plane perpendicular to the magnetic field vector [18]. The accuracy of satellite attitude control systems (ACS) using only magnetic actuators is known to be accurate on the order of $0.4-0.5$ degree [18]. The satellite cannot be controlled precisely in three-dimensional space using only magnetorquers [18], but the combination of magnetorquers with one reaction wheel expands the two-dimensional control torque possibilities to be three-dimensional. The attitude accuracy of the combined actuators has been compared with three reaction wheels-based attitude control in the references $[18,19]$. Classical sliding mode control has also been used for magnetic actuated spacecraft $[20,21]$. However, the proposed nonlinear adaptive fuzzy sliding mode control law has never been used in magnetic attitude control.

To address the attitude tracking problem, the attitude tracking error $q_{e}=\left(\bar{q}_{e}^{T}, q_{4 e}\right)^{T}$ is defined as the relative orientation between the body frame and the desired frame with orientation $q_{d}=\left(\bar{q}_{d}^{T}, q_{4 d}\right)^{T}$. In order to apply the proposed nonlinear controller, the equations of motion are rewritten as

$$
\ddot{q}_{e}=f\left(\bar{q}_{e}, \dot{\bar{q}}_{e}\right)+\widehat{\tau}_{c}+\widehat{\tau}_{m}+\widehat{\tau}_{d} .
$$

The adaptive fuzzy sliding mode magnetic control law is given by

$$
\begin{gathered}
\tau=-k_{1} S-\theta^{T} \xi-k_{2} \tanh \left(\frac{3 K_{u} \varsigma S}{\epsilon}\right), \\
\dot{\theta}=\delta S \xi \theta, \\
\tau_{\text {ap }}=\frac{\tau S}{\left\|S^{2}\right\| S}, \\
M=\tau_{\text {ap }} \times \frac{B}{\left\|B^{2}\right\|} .
\end{gathered}
$$

Here, $\widehat{\tau}_{m}=M \times B$ are the torques generated by the magnetorquers, $M$ is the vector of magnetic dipoles for the three magnetorquers, and $B$ is the vector formed with the components of the Earth's magnetic field in the body frame of the reference. $S=\dot{\bar{q}}_{e}+K \bar{q}_{e}$ is the sliding surface. $\theta$ and $\xi$ are the adaptive parameters and fuzzy weight functions generated by the fuzzy logic systems [22], and $\delta, k_{1}, k_{2}, K_{u}, \varsigma, \epsilon$ are positive constants used for tuning the control response.

Remark 1. AFSMC controller design details can be found in the author's previous papers [11]. The design includes: (1) Sliding surface design [22] and (2) fuzzy logic system design [22].

4.2. Simulation Results. The attitude detumbling and attitude stabilization phases are considered in the ACS simulation. The B-dot algorithm, PD magnetic control law, and adaptive fuzzy sliding mode magnetic control law are used for the two phases, respectively. We note that the orbit used for the present simulation study is a $500 \mathrm{~km}$ circular orbit with $45^{\circ}$ inclination. At this altitude, the total disturbance torque for $1 \mathrm{U}$ CubeSats is estimated to be on the order of $5 \times 10^{-7} \mathrm{Nm}$. This is intended to be a slight overestimation to include a safety margin.

\subsubsection{Detumbling Mode and Stabilization Mode}

Scenario 1. In the initial stage of ACS control, the angular velocities of the satellite are assumed to be $0.169 \mathrm{rad} / \mathrm{s}$ as a result of separation from the launch vehicle. The ACS damps the angular rate by controlling three magnetorquers. The control logic generally used for detumbling is called B-dot 


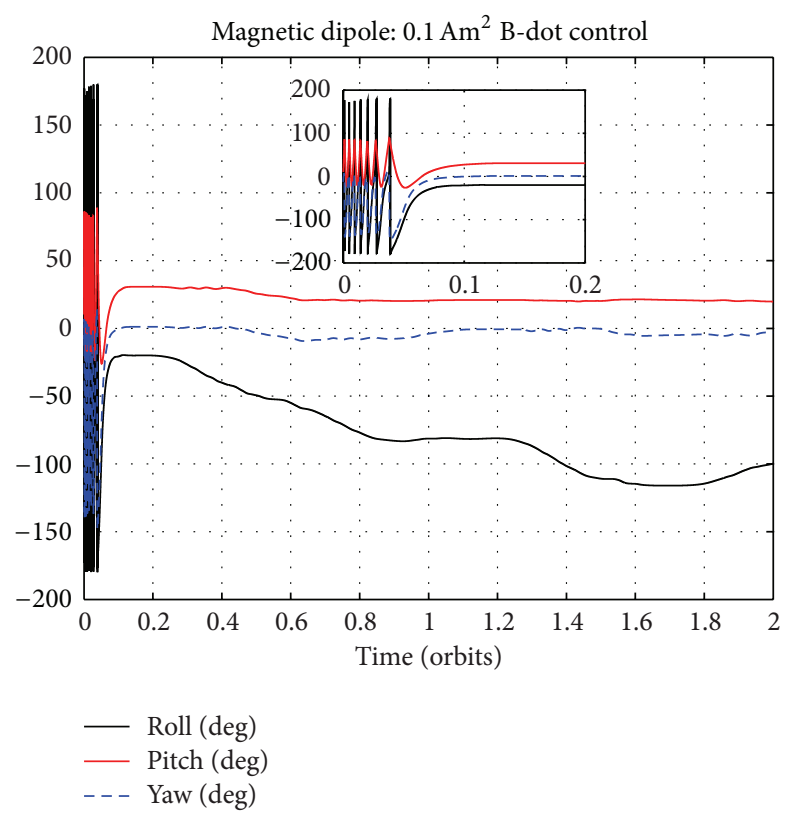

(a)

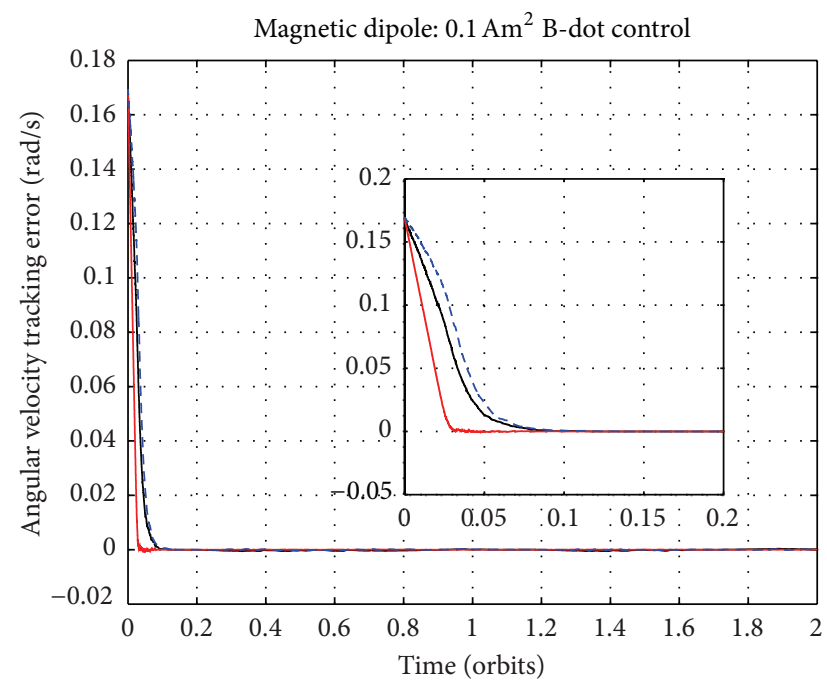

(c)

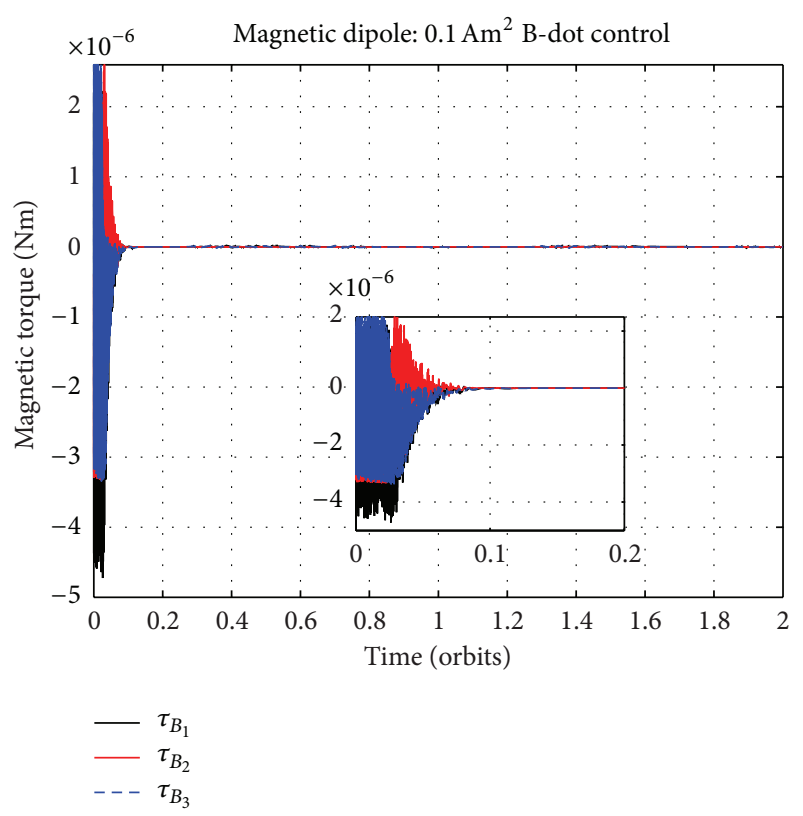

(b)

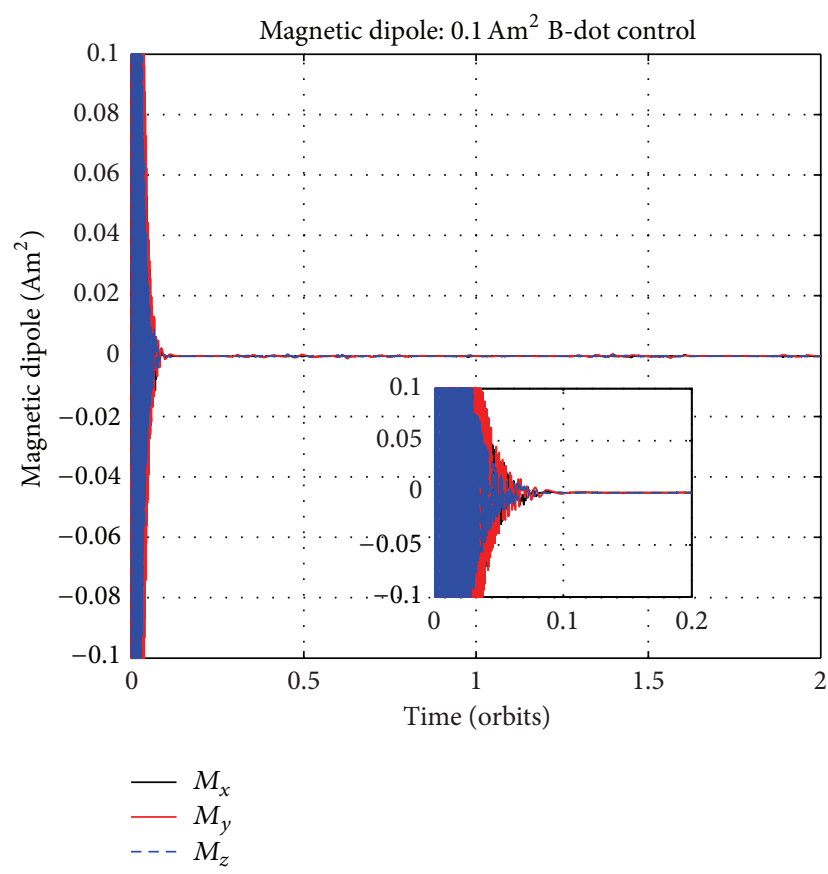

(d)

FIgURE 7: Scenario 1 detumbling control results.

control [1], as it makes use of the derivative of the magnetic field " $B$ ". For a CubeSat with moment of inertia $J=$ $\operatorname{diag}(0.002,0.002,0.002) \mathrm{kg} \mathrm{m}^{2}$, we include the external disturbances (aerodynamic, gravity gradient, and solar pressure), set the desired quaternion to be $(0,0,0,1)$, set the initial quaternion to be $(0.1,-0.1,0.1,0.9849)$, and assume the magnetic dipole maximum of the rods to be $0.1 \mathrm{Am}^{2}$. The Euler angle tracking errors, angular velocity tracking errors, and magnetic torquers, magnetic dipoles results are shown in Figure 7. The satellite starts at the selected tip-off rate, and, after 1 orbit, the angular velocities are reduced to the required rates before continuing with other ACS tasks.

Scenario 2. Now, we consider a CubeSat with the same moment of inertia and orbit and assume the magnetic dipole maximum of the magnetorquer to be $0.4 \mathrm{Am}^{2}$ with a magnetometer sensor bias calculated by $20 * 10^{-4} * \operatorname{rand}(1)$. Proportional-derivative (PD) magnetic control [23] laws (shown in (15)) are used in this simulation and the results over 6 orbits are shown in Figure 8. During the first three orbits, three 


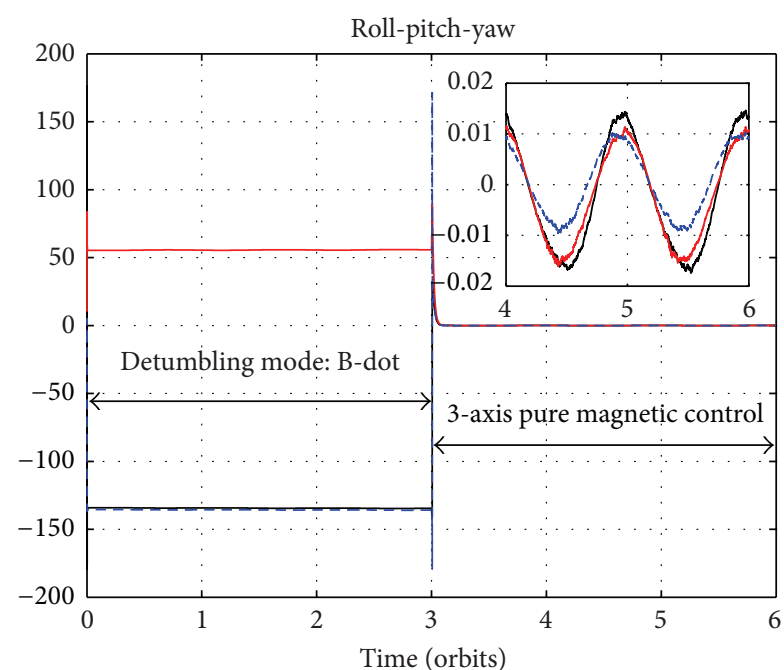

(a)

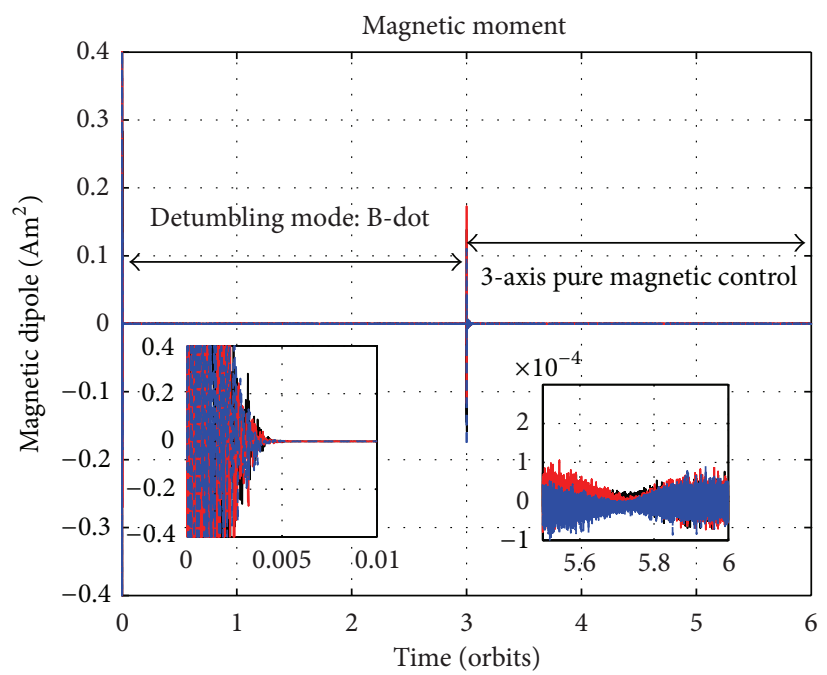$$
\begin{array}{r}
-M_{x} \\
-M_{y} \\
---M_{z}
\end{array}
$$

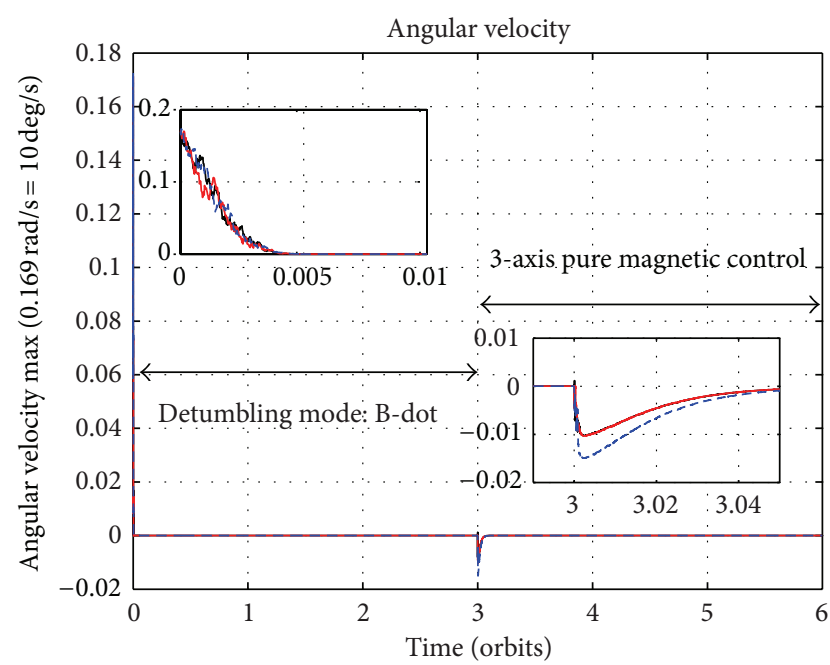

(b)

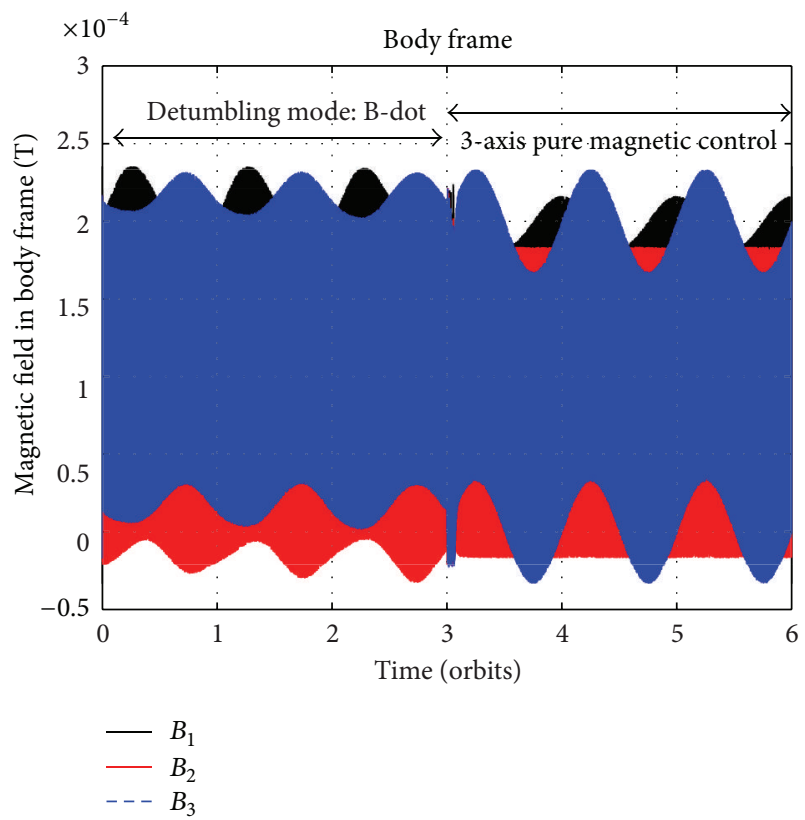

(d)

(c)

Figure 8: Scenario 2 detumbling mode and stable mode control results.

magnetorquers are used for the detumbling mode. In the second three orbits, three magnetorquers and one reaction wheel are used for the stable mode. The attitude control accuracy is less than 0.02 degree while using the PD magnetic control laws

$$
\begin{gathered}
\widehat{\tau}_{m}=M \times B, \\
M=K_{1} \omega_{b} \times B, \\
M=K_{1} \omega_{b} \times B+K_{2} q \times B .
\end{gathered}
$$

4.2.2. Attitude Stabilization Mode: Nadir and Limb Pointing with Three Magnetorquers and One Pitch Reaction Wheel. Next, we consider the second stage of nanosatellite control with a low initial angular velocity after detumbling and large slew angle target for limb and nadir pointing. The configuration with three magnetorquers and one flywheel [24] has been used for many years. In a real nanosatellite mission, hardware failures of the reaction wheels are very common [19]. When there are two wheel failures, the ACS can be switched from using three reaction wheels to three magnetorquers and one reaction wheel, and attitude control accuracy maintained using this method. The adaptive fuzzy sliding mode magnetic control laws are shown in (11)-(14). The attitude control accuracy using the nonlinear adaptive fuzzy sliding mode control law will be more robust to the external disturbances than using the PD magnetic control law in (15).

Scenario 3. An ACS using one reaction wheel and three magnetorquers as actuators for limb pointing with the desired 


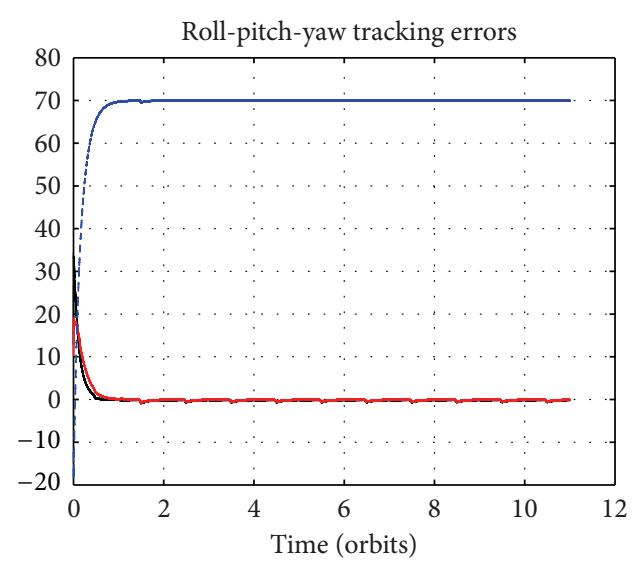

(a)

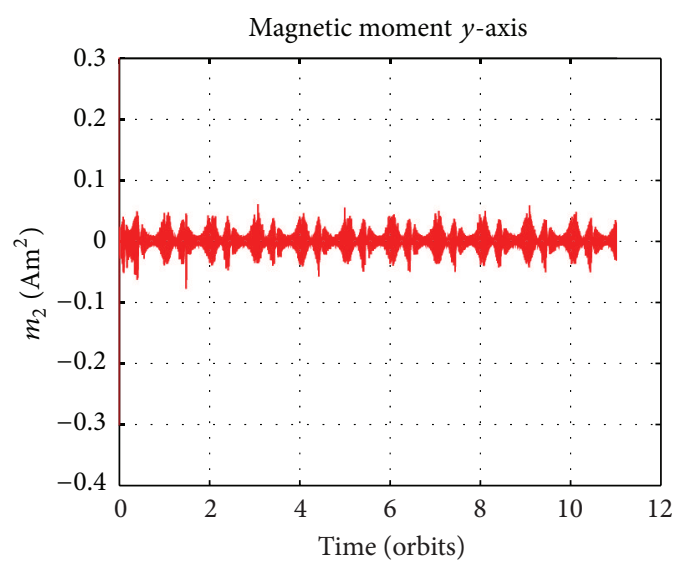

(c)

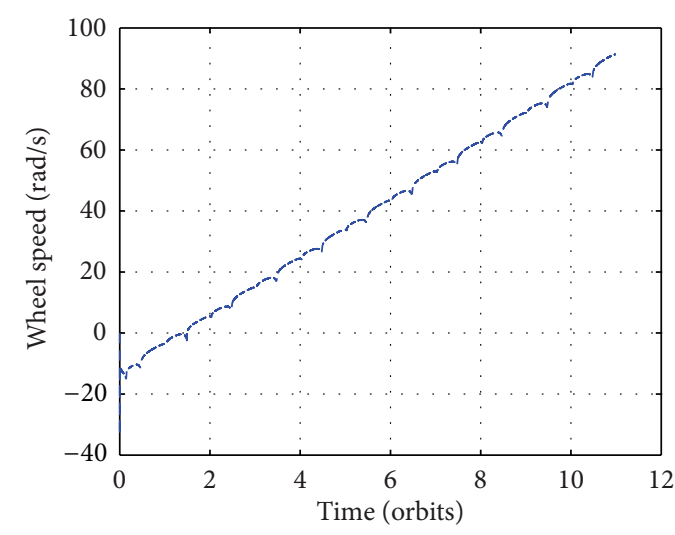

(e)

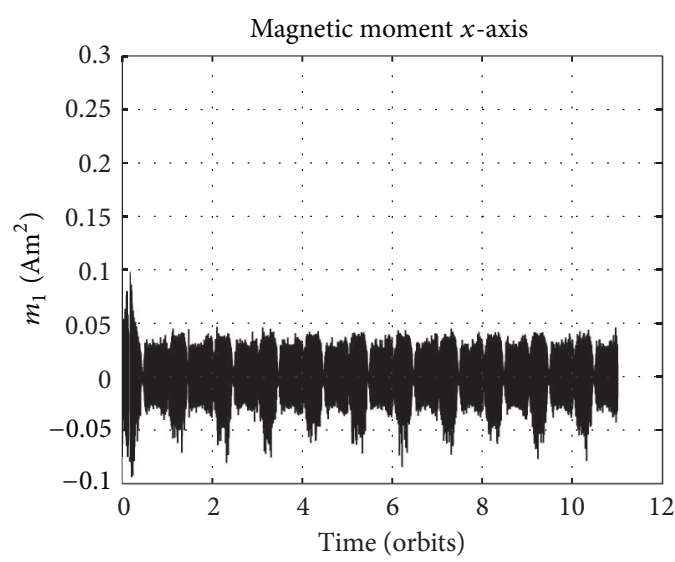

(b)

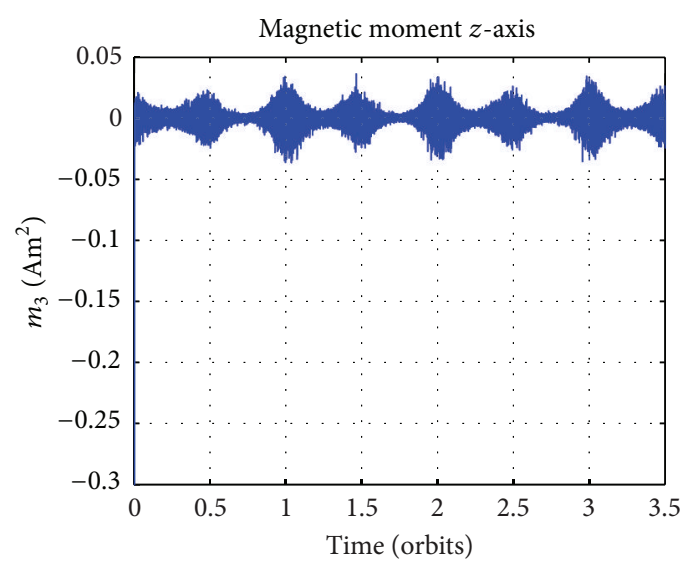

(d)

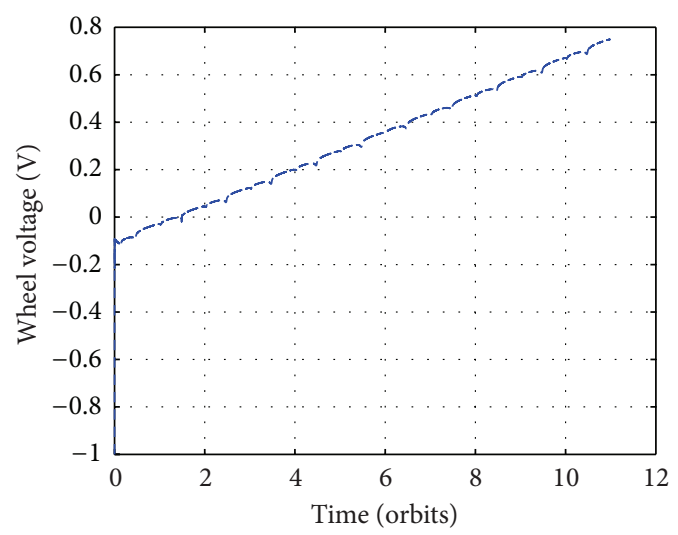

(f)

FIGURE 9: Scenario 3 limb pointing control results using one reaction wheel and three magnetorquers.

quaternion set to $(0,0.5736,0,0.8192)$ is examined using AFSMC over 10 orbits. The wheel dead zone is not considered here. The initial quaternion is $(0.1,-0.1,0.1,0.9849)$ and initial angular velocity is $(0.0169,0.0169,0.0169) \mathrm{rad} / \mathrm{s}$. Considering only the pitch reaction wheel is available, the numerical simulations demonstrate that the proposed technique achieves a high pointing accuracy $(<0.09$ degree) for small satellites. The magnetic dipoles from the three magnetorquers, attitude tracking errors, wheel speed, and wheel voltage are shown in Figure 9.

Scenario 4. An ACS using one reaction wheel and three magnetorquers as actuators for nadir pointing with the desired quaternion that is set to $(0,0,0,1)$ is examined using AFSMC over 10 orbits. The wheel dead zone is assumed to be $\pm 1.0 \mathrm{~V}$. The initial quaternion is $(0.1,-0.1,0.1,0.9849)$ and initial 


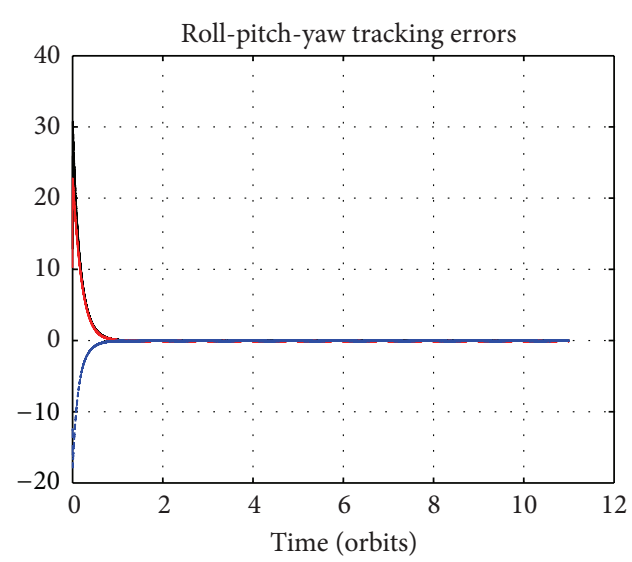

(a)

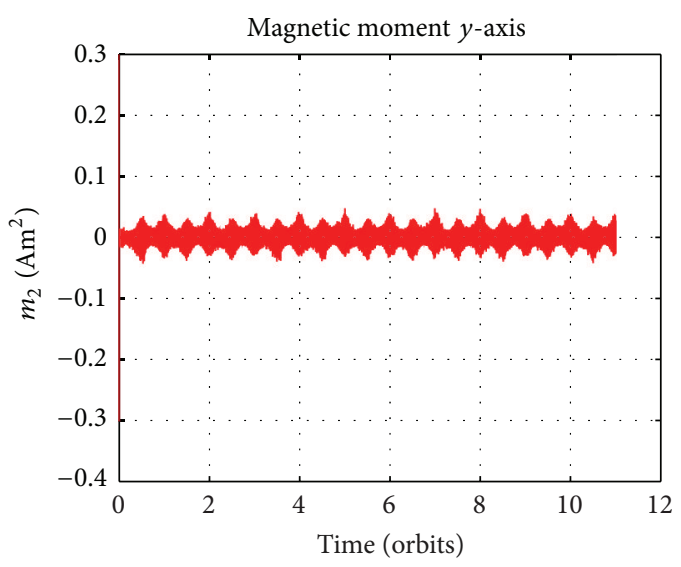

(c)

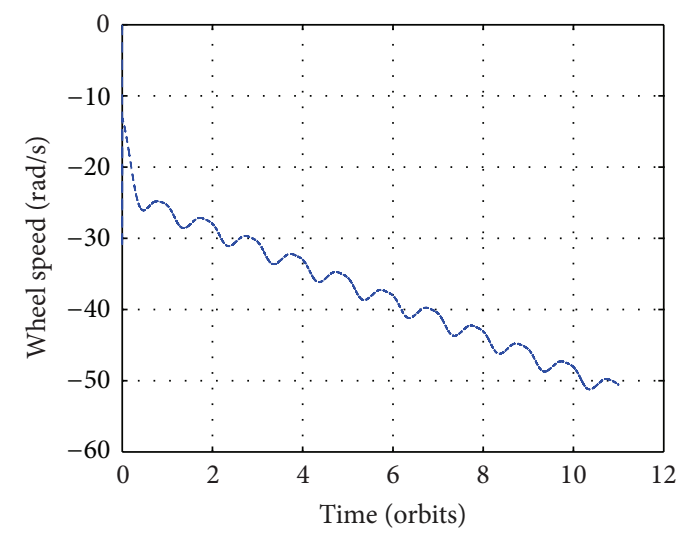

(e)

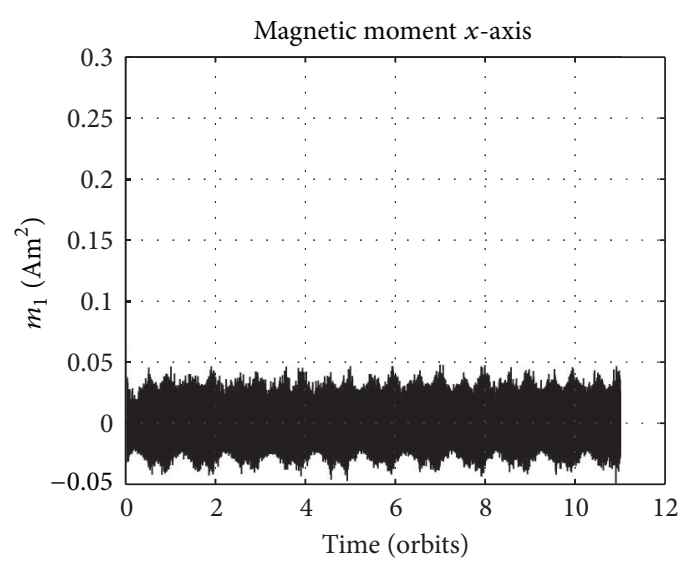

(b)

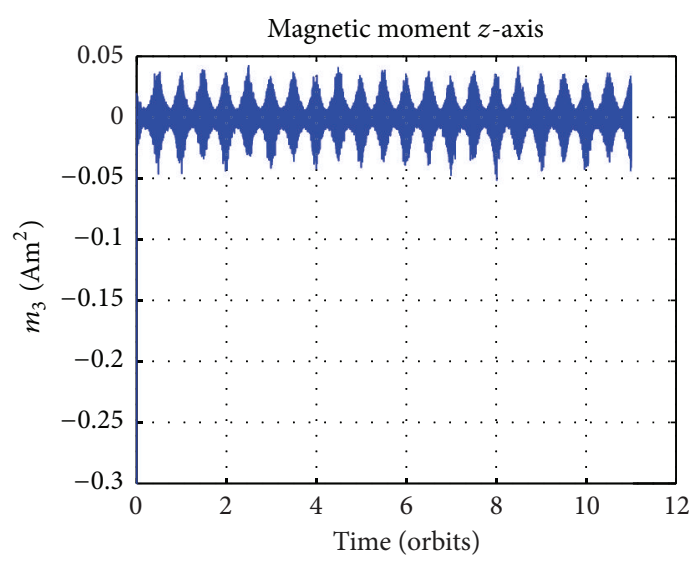

(d)

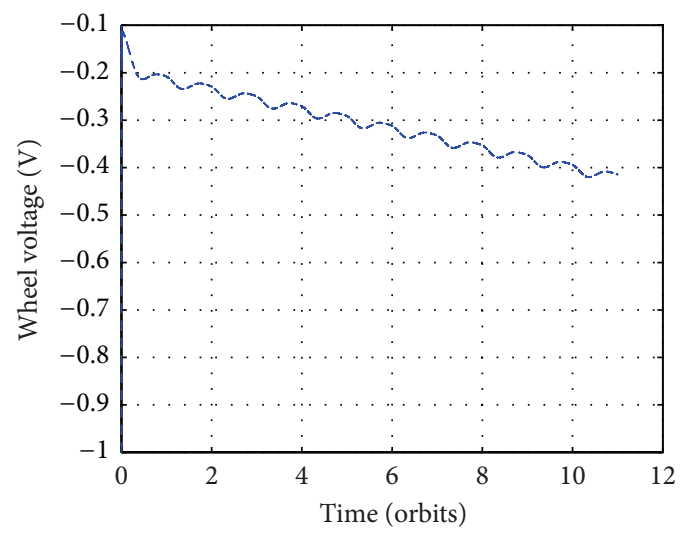

(f)

FIGURE 10: Scenario 4 nadir pointing control results using one reaction wheel and three magnetorquers.

angular velocity is $(0.0169,0.0169,0.0169) \mathrm{rad} / \mathrm{s}$. The pitch reaction wheel and three magnetorquers are used. These simulations demonstrate that the proposed technique can also achieve high accuracy (<0.09 degree with all disturbances) pointing control for small satellites. The magnetic dipoles of the three magnetorquers, attitude tracking errors, wheel speed, and wheel voltage are shown in Figure 10. It takes about 40 orbits for the wheel speed to increase from 0 to $2000 \mathrm{rpm}$ (209 rad/s).

\subsubsection{Attitude Stabilization Mode: Nadir Pointing with} Three Reaction Wheels

Scenario 5. An ACS using three reaction wheels as actuators for nadir pointing with the desired quaternion set to $(0,0,0,1)$ is examined using AFSMC over 0.1 orbits. The wheel dead zone is assumed to be $\pm 1.0 \mathrm{~V}$. The initial quaternion is $(0.1,-0.1,0.1,0.9849)$ and initial angular velocity is $(0.0169,0.0169,0.0169) \mathrm{rad} / \mathrm{s}$. This configuration can also 


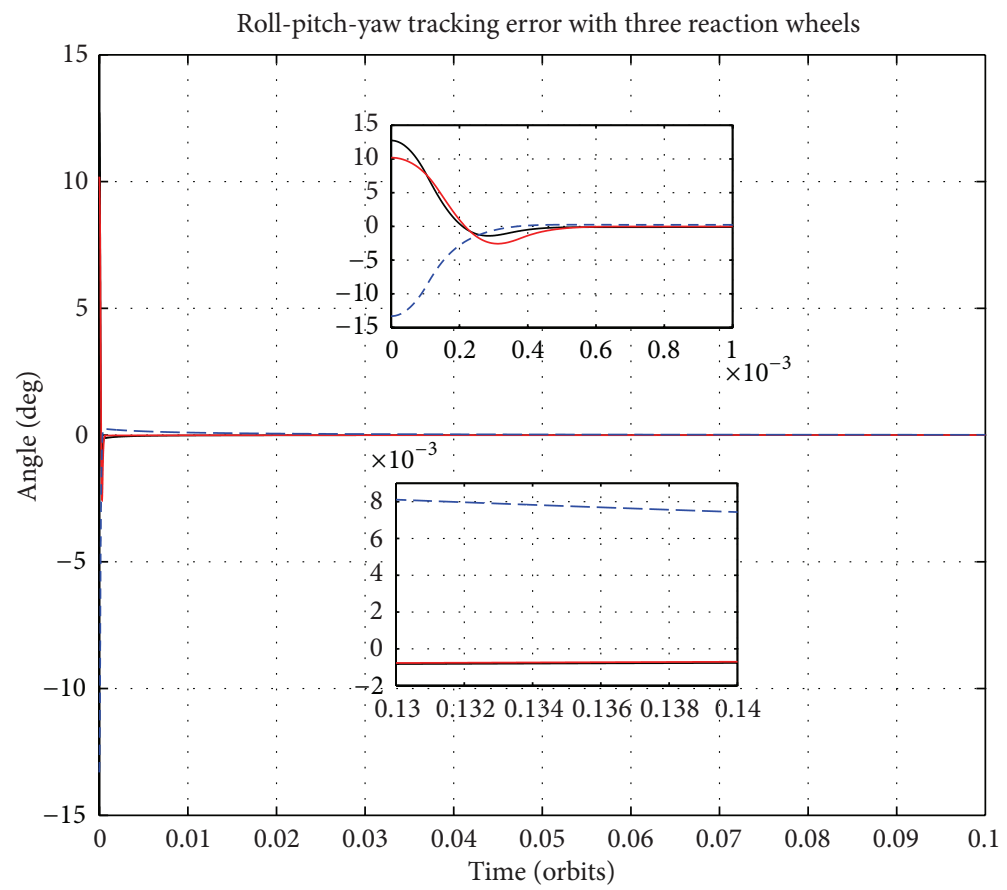

FIGURE 11: Scenario 5 nadir pointing control results using three wheels: Euler angle tracking errors.

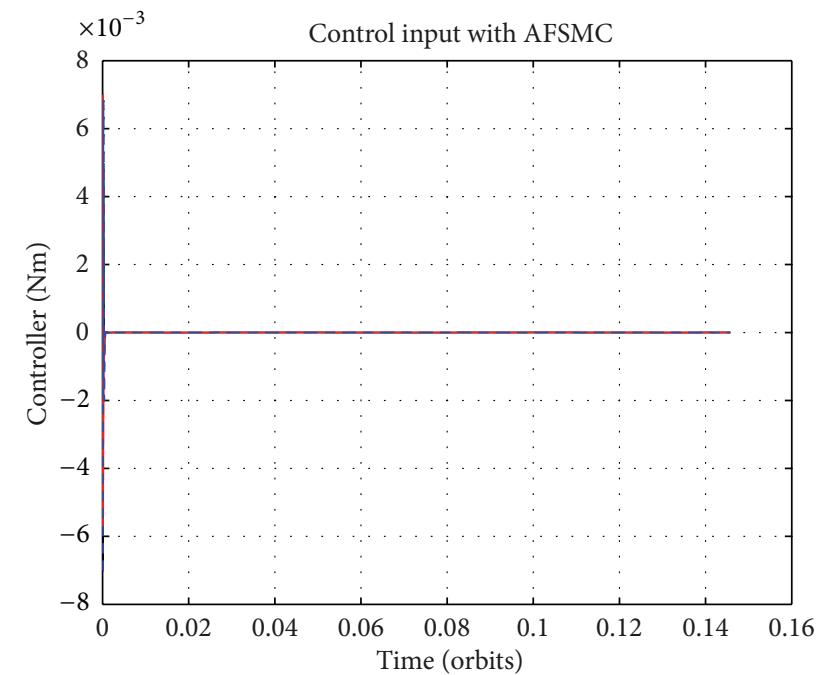

FIGURE 12: Scenario 5 nadir pointing control results using three wheels: control input.

achieve high attitude control accuracy $(<0.008$ degree with all disturbances). The reaction wheel attitude control laws use (11) and (12). The settling time is shorter and the pointing accuracy is higher than that achieved using only three magnetorquers and one reaction wheel as actuators. However, the power consumption is higher than using three magnetorquers and one reaction wheel. The Euler angle tracking errors, control inputs, wheel speeds, and wheel voltages are shown in Figures 11, 12, 13, and 14 .

\section{Satellite Attitude Control System Hardware Testing}

5.1. Spherical Air-Bearing Testbed for Satellite Attitude Control Systems. The ground testing of the proposed CubeSat ACS design was performed at York University using a nanosatellite attitude control testbed. This facility consists of a spherical air-bearing platform $[25,26]$ suspended upon a thin layer of air providing a full three degrees of freedom with negligible friction for ACS testing. The platform includes a manual balancing system and platform electronics that include an on-board computer (OBC), wireless transceiver for telemetry, reference inertial measurement unit (IMU), power distribution board, and batteries. The air-bearing system used for $1 \mathrm{U}$ CubeSat testing is shown in Figure 15.

5.2. Ground Test Results: ACS Testing Results with Three Reaction Wheel Actuators. Nonlinear attitude control has been explored widely in theory $[14,27,28]$. In real satellite applications, nonlinear controllers are usually not selected due to their complexity of design. We have tested nonlinear control algorithms [11] with our spherical air-bearing system. We now test the proposed control method (from (11) and (12)) on this system using the sensors and ACS board described above and three-axis reaction wheel actuation. A PID control law is also used for comparison on the same hardware. The control laws are programmed in the $\mathrm{C}$ language, and the $\mathrm{OBC}$ runs the Linux operating system. More details of the implementation can be also found in [11]. Here, we will show air-bearing system test results with a $1 \mathrm{U}$ CubeSat. The design parameters of the control laws used are given in Tables 3 and 4 . 


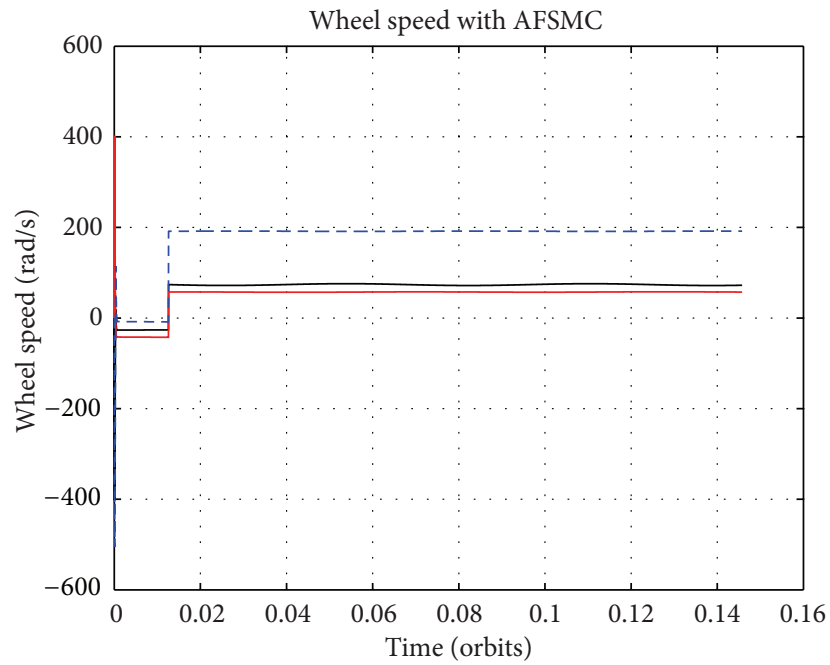

FIGURE 13: Scenario 5 nadir pointing control results using three wheels: wheel speed.

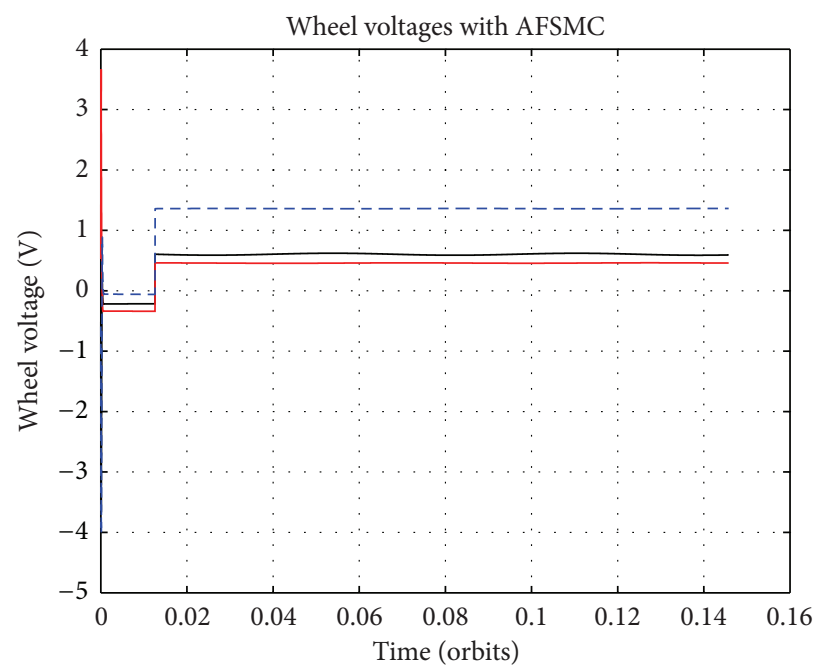

FIGURE 14: Scenario 5 nadir pointing control results using three wheels: wheel voltage.
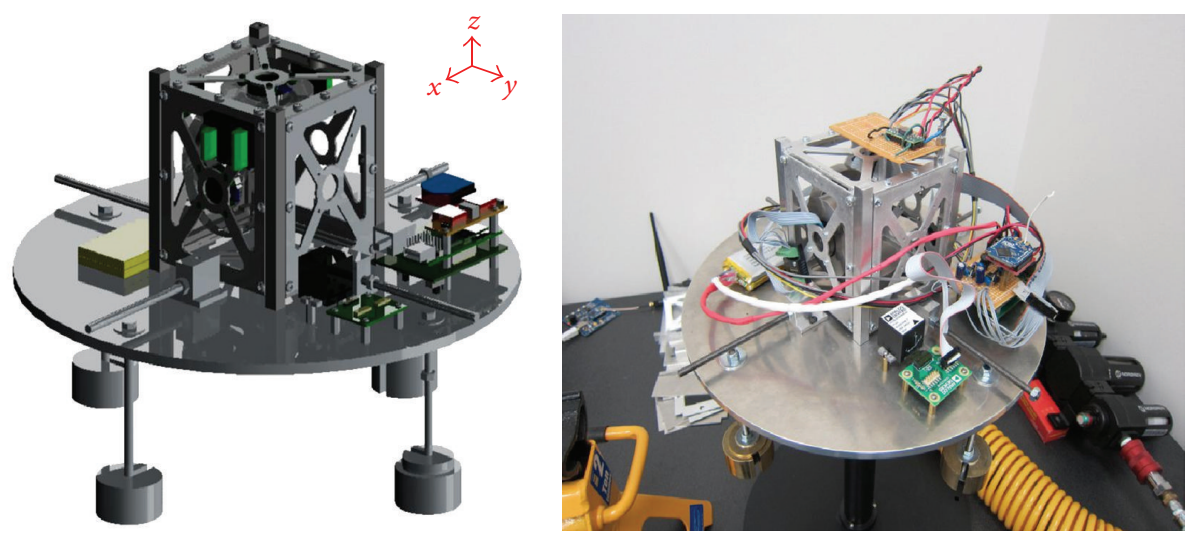

FIGURE 15: Air-bearing ACS ground testing system. 


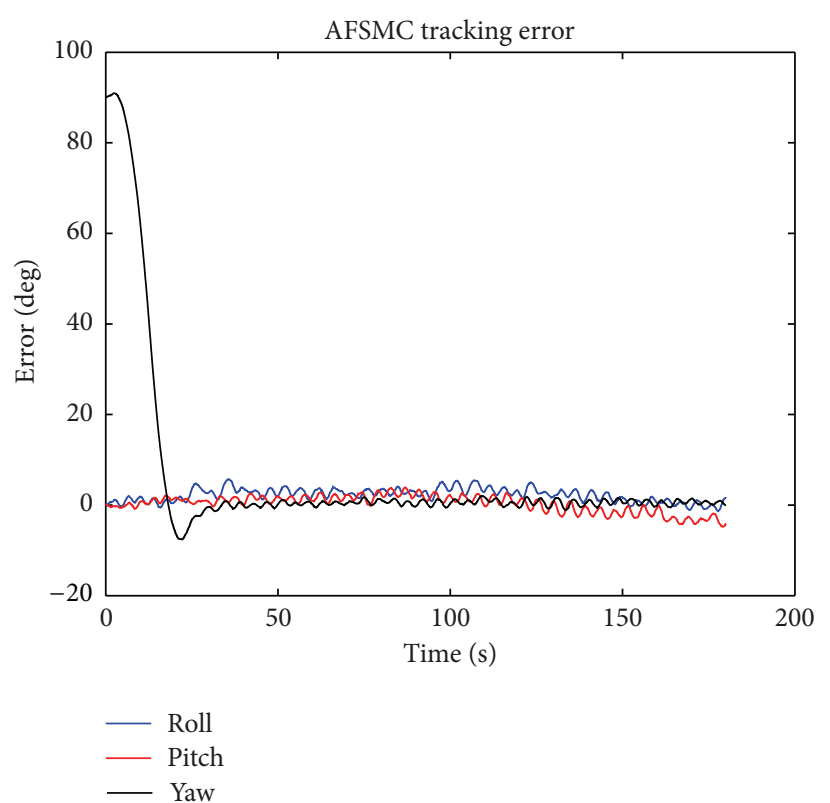

(a)

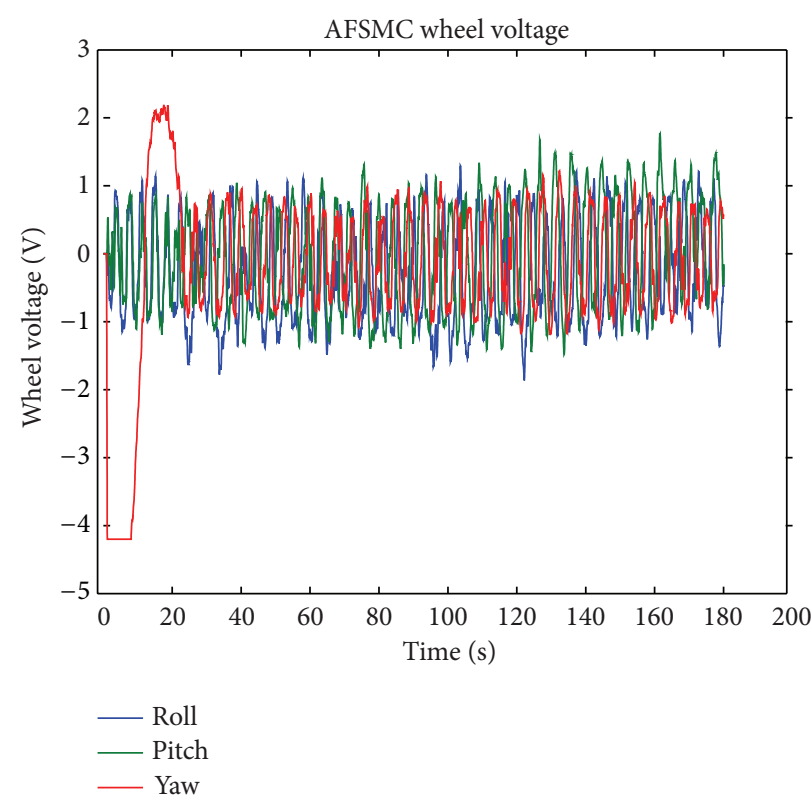

(c)

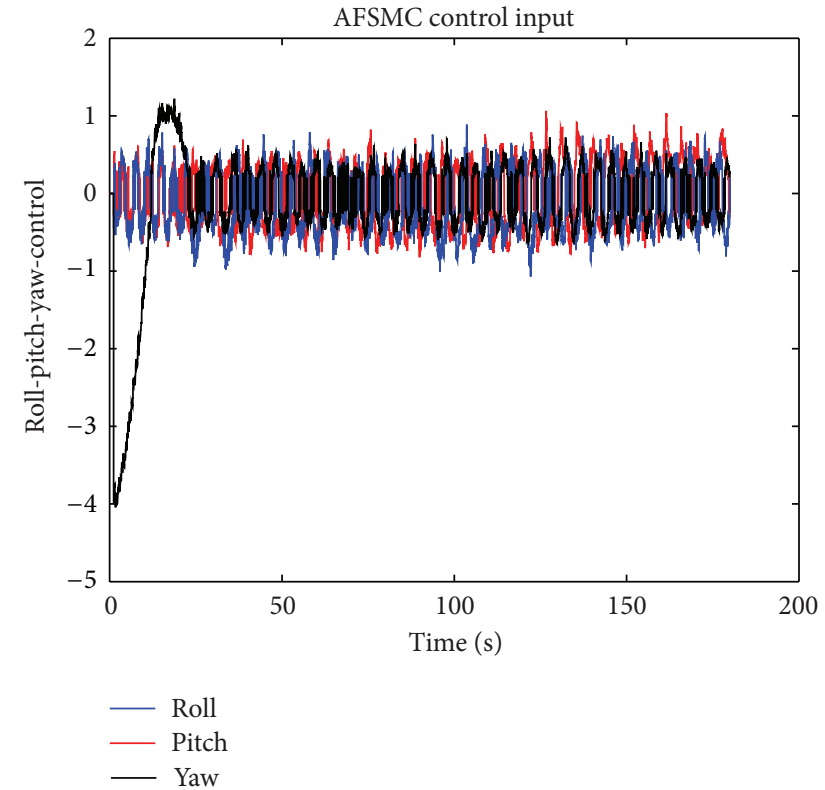

(b)

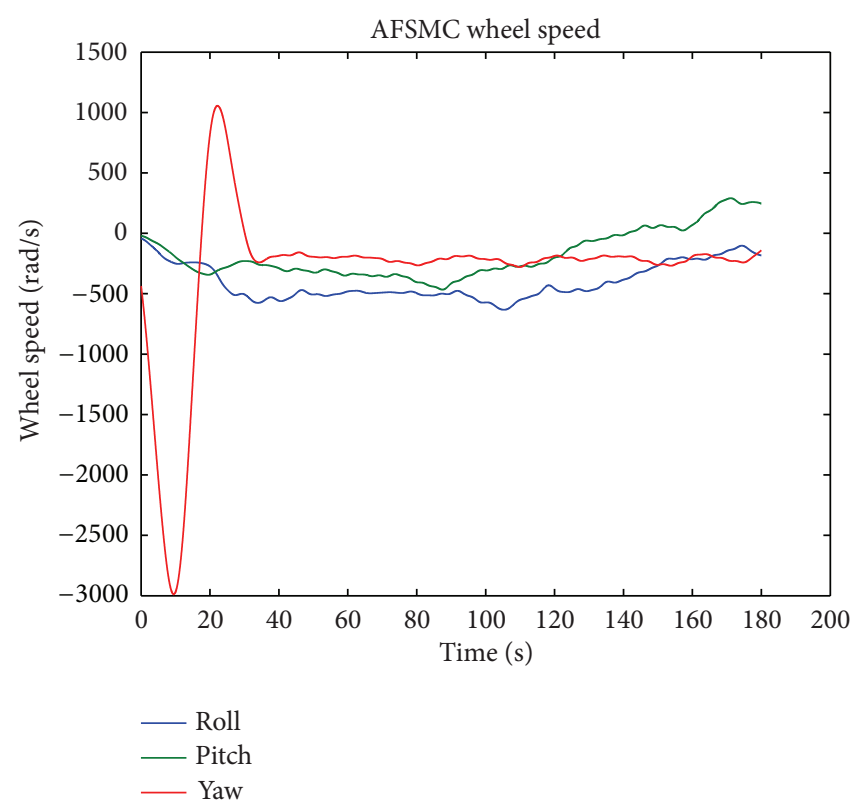

(d)

FIGURE 16: Air-bearing AFSMC controller results.

TABLE 3: PID controller parameters for air bearing testing.

\begin{tabular}{lc}
\hline Name & Values \\
\hline Proportional parameters & 0.03 \\
Integral parameters & 0.0001 \\
Derivative parameters & 0.11 \\
\hline
\end{tabular}

Figures 16 and 17 show the attitude tracking errors, AFSMC/PID control output signals, reaction wheel voltages, and reaction wheel velocities for a 90 degree yaw slew of the system about the $z$-axis while maintaining 0 degrees of roll
TABLE 4: AFSMC controller parameters for air bearing testing.

\begin{tabular}{lc}
\hline Name & Values \\
\hline Sliding surface gain & $0.00001,100$ \\
Fuzzy membership function & 1,1 \\
Adaptive gains $\delta, K_{u}, \varsigma, \epsilon$ & $0.1,0.36,0.01,2$ \\
\hline AFSMC parameter $k_{1}$ & 0.004 \\
\hline AFSMC parameter $k_{2}$ & 0.0025 \\
\hline
\end{tabular}

and pitch. Due to the difficulty of perfectly balancing the air-bearing system, a gravitational disturbance, larger than 


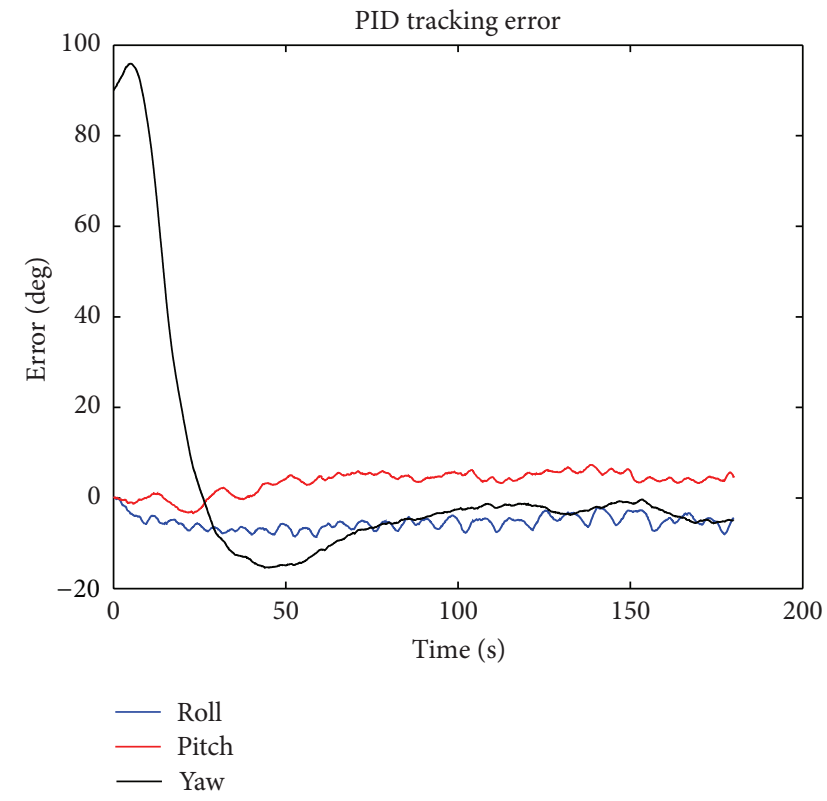

(a)

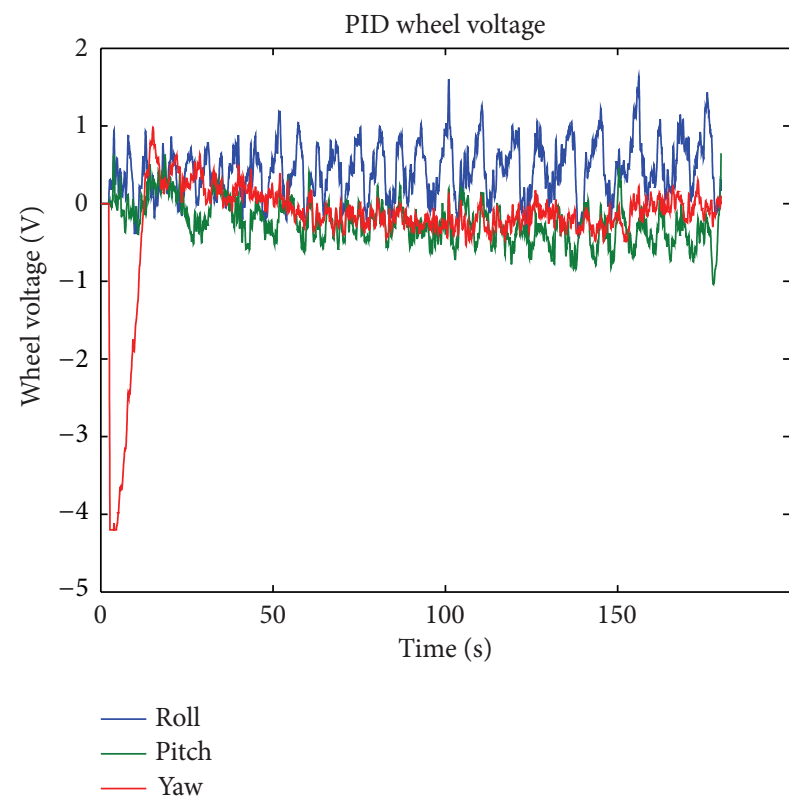

(c)

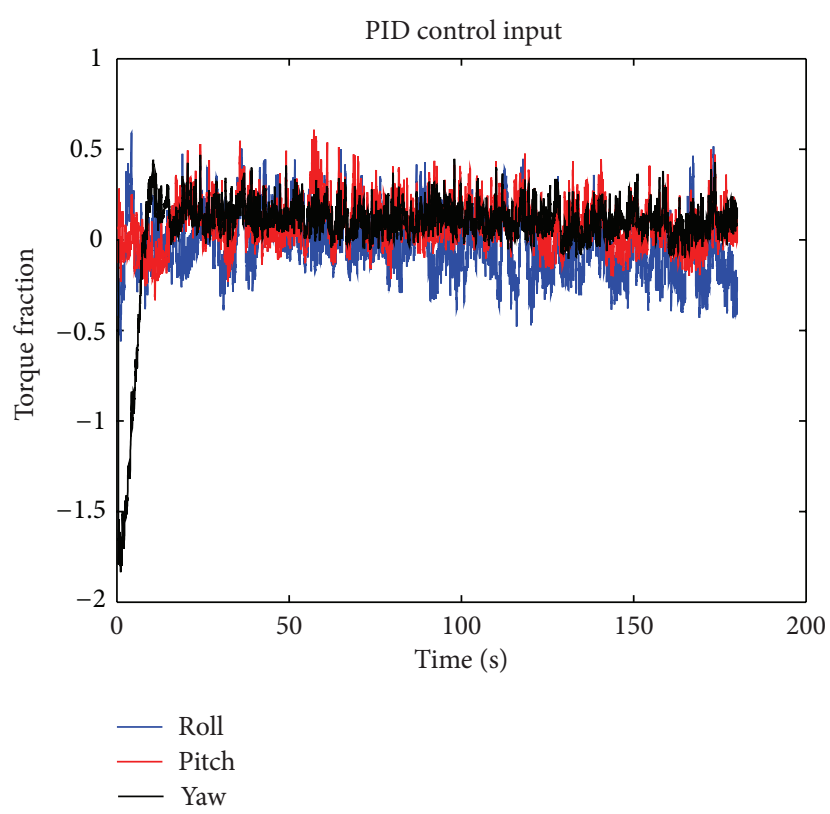

(b)

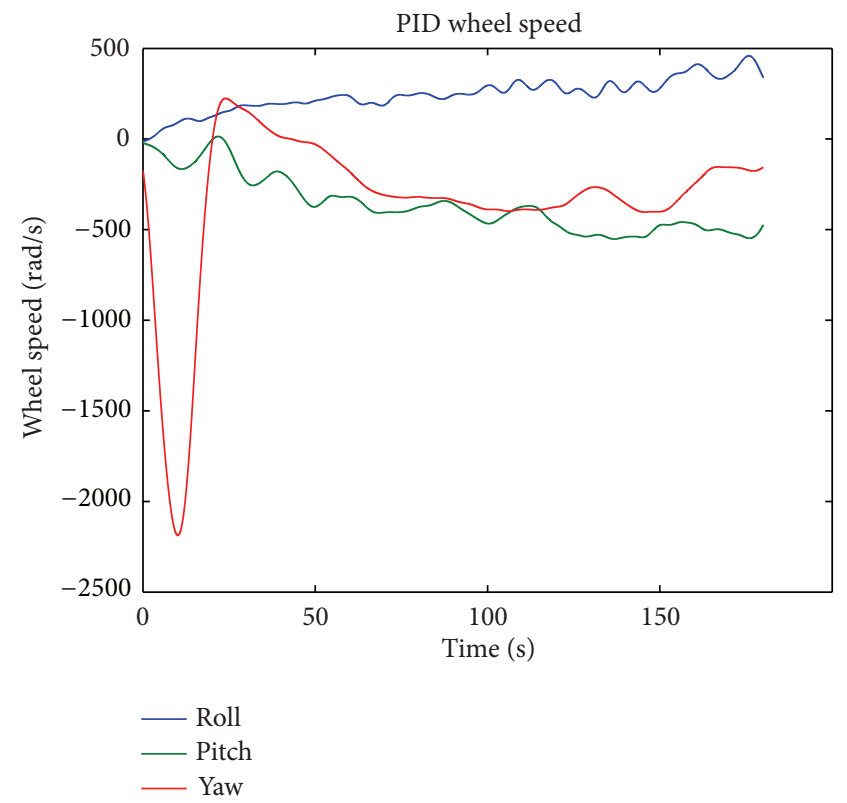

(d)

FIGURE 17: Air-bearing PID controller results.

normal in size, is considered to be present about the $x$ and $y$ rotational axes. Compared to the PID controller, the AFSMC controller uses nearly the same gain and has much better tracking performance under these conditions.

\section{Future Work}

While the ground test demonstrates the effective and promising control accuracy of the proposed ACS design, it is difficult to predict the performance of pure and hybrid magnetic control as the Earth's magnetic field present in the test facility is constant and invariant with many sources of additional magnetic noise. The next step in the development of a complete ACS test facility is the addition of a Helmholtz cage for magnetic control tests. The magnetic cage and the airbearing system in the lab are shown in Figure 18 and are presently being prepared for air-bearing magnetic control testing. Future work will demonstrate the effectiveness of magnetic control in further ground testing. 


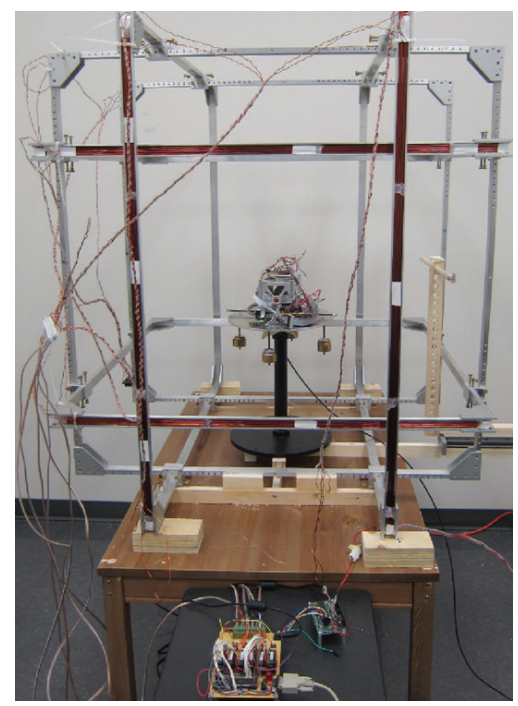

FIGURE 18: Helmholtz cage for magnetic field generation.

\section{Conclusion}

In this paper, the proposed attitude control system design will allow inexpensive and capable satellites to be developed for academic use. The proposed attitude control system uses three reaction wheels as actuators or one wheel with three magnetorquers as actuators. The hardware designs of the actuators and embedded attitude control systems are also described for prototype development. Five different scenarios of numerical simulation results for a $1 \mathrm{U}$ CubeSat-class nanosatellite show the effectiveness of the proposed attitude control design for detumbling and attitude pointing purposes. The ground testing results in this paper provide a promising comparison of an adaptive fuzzy sliding mode controller with a conventional proportional-integral-derivative controller for a $1 \mathrm{U}$ CubeSat-class nanosatellite. The nanosatellite testing hardware can be also extended for use in $2 \mathrm{U}$ or $3 \mathrm{U}$ CubeSatclass nanosatellites in the future.

\section{Acknowledgments}

The authors gratefully acknowledge the support provided by COM DEV Ltd., NSERC, MITACS, and OCE. The authors would also like to acknowledge the work of M. Cannata, I. Proper, T. Ustrzycki, G. Benari, and H. Hakima in this paper.

\section{References}

[1] Y. W. Jan and J. C. Chiou, "Attitude control system for ROCSAT3 microsatellite: a conceptual design," Acta Astronautica, vol. 56, no. 4, pp. 439-452, 2005.

[2] M. Ovchinnikov, V. Pen'kov, O. Norberg, and S. Barabash, "Attitude control system for the first Swedish nanosatellite 'MUNIN"' Acta Astronautica, vol. 46, no. 2, pp. 319-326, 2000.

[3] M. I. Martinelli and R. S. S. Peña, "Passive 3 axis attitude control of MSU-1 pico-satellite," Acta Astronautica, vol. 56, no. 5, pp. 507-517, 2005.
[4] G. P. Candini, F. Piergentili, and F. Santoni, "Miniaturized attitude control system for nanosatellites," Acta Astronautica, vol. 81, pp. 325-334, 2005.

[5] T. Xiang, T. Meng, H. Wang, K. Han, and Z. H. Jin, "Design and on-orbit performance of the attitude determination and control system for the ZDPS-1A pico-satellite," Acta Astronautica, vol. 77, pp. 82-196, 2012.

[6] M. Abdekrahman and S. Y. Park, "Integrated attitude determination and control system via magnetic measurements and actuation," Acta Astronautica, vol. 69, no. 3-4, pp. 168-185, 2011.

[7] M. Cannata, Development of a sun vector determination algorithm for Cubesat-Class spacecraft [M.S. thesis], Dept. of Earth and Space Science and Engineering, York University, Toronto, Canada, 2010.

[8] I. Proper, Reaction wheel design, construction and qualification testing [M.S. thesis], Dept. of Earth and Space Science and Engineering, York University, Toronto, Canada, 2010.

[9] J. Li, M. A. Post, and R. Lee, "Real time fault tolerant nonlinear attitude control system for nanosatellite application," in AIAA Infotech@Aerospace 2012 Conference, pp. 19-21, Garden Grove, Calif, USA, 2012.

[10] J. Li, M. A. Post, and R. Lee, "Nanosatellite air bearing tests of fault-tolerant sliding-mode attitude control with Unscented Kalman Filter," in AIAA Guidance, Navigation, and Control Conference, Minnesota, Minneapolis, Minn, USA, 2012.

[11] J. Li, M. A. Post, and R. Lee, "Nanosatellite attitude air bearing system using variable structure control," in IEEE 25th Annual Canadian Conference on Electrical and Computer Engineering, Montreal, Canada, May 2012.

[12] M. F. Mehrjardi and M. Mirshams, "Design and manufacturing of a research magnetic torquer Rod," Contemporary Engineering Sciences, vol. 3, no. 5, pp. 227-236, 2010.

[13] T. Ustrzycki, Spherical air bearing testbed for nanosatellite attitude control development [M.S. thesis], Dept. of Earth and Space Science and Engineering, York University,, Toronto, Canada, 2011.

[14] C. H. Won, "Comparative study of various control methods for attitude control of a LEO satellite," Aerospace Science and Technology, vol. 5, pp. 323-333, 1999.

[15] J. Y. Lin, S. Ko, and C. K. Ryoo, "Fault tolerant control of satellites with four reaction wheels," Control Engineering Practice, vol. 16, no. 10, pp. 1250-1258, 2008.

[16] E. Silani and M. Lovera, "Magnetic spacecraft attitude control: a survey and some new results," Control Engineering Practice, vol. 13, no. 3, pp. 357-371, 2005.

[17] C. J. Dameran, "Hybrid magnetic attitude control gain selection," Proceedings of the Institution of Mechanical Engineers G, vol. 223, no. 8, pp. 1041-1047, 2009.

[18] Z. Q. Zhou, "Spacecraft attitude tracking and maneuver using combined magnetic actuators," in AIAA Guidance, Navigation, and Control Conference, Toronto, Ontario, Canada, August 2010.

[19] J. R. Forbes and C. J. Damaren, "Geometric approach to spacecraft attitude control using magnetic and mechanical actuation," Journal of Guidance, Control, and Dynamics, vol. 33, no. 2, pp. 590-595, 2010.

[20] R. Wisniewski, Satellite attitude control using magnetic actuation only [Ph.D. thesis], Dissertation, Dept. of Control Engineering, Aalborg University, Denmark, 1996. 
[21] P. Wang, Y. B. Shtessel, and Y. Q. Wang, "Satellite attitude control using only magnetorquers," in Proceeding of the 13th Southeastern Symposium on System Theory, West Virginia University, Morgantown, WVa, USA, March 1998.

[22] J. Li and K. D. Kumar, "Fault tolerant attitude synchronization control during formation flying," Journal of Aerospace Engineering, vol. 24, no. 3, pp. 251-263, 2011.

[23] D. V. Guerrant, Design and analysis of fully magnetic control for picosatellite stabilization [M.S. thesis], California Polytechnic State University, 2005.

[24] M. Chen, S. J. Zhang, F. R. Liu, and Y. C. Zhang, "Combined attitude control of small satellite using One flywheel and magnetic torquers," in Proceedings of the 2nd International Symposium on Systems and Control in Aerospace and Astronautics (ISSCAA '08), Shenzhen, China, December 2008.

[25] J. Prado, G. Bisiacchi, L. Reyes et al., "Three-axis air-bearing based platform for small satellite attitude determination and control simulation," Journal of Applied Research and Technology, vol. 3, no. 3, pp. 222-237, 2005.

[26] J. J. Kim and B. N. Agrawal, "Automatic mass balancing of airbearing-based three-axis rotational spacecraft simulator," AIAA Journal of Guidance, Control, and Dynamics, vol. 32, no. 3, pp. 1005-1017, 2009.

[27] K. S. Kim and Y. Kim, "Robust backstepping control for slew maneuver using nonlinear tracking function," IEEE Transactions on Control Systems Technology, vol. 11, no. 6, pp. 822-829, 2003.

[28] S. C. Lo and Y. P. Chen, "Smooth sliding mode control for spacecraft attitude tracking maneuvers," Journal of Guidance Control and Dynamics, vol. 18, no. 6, pp. 1345-1349, 1995. 

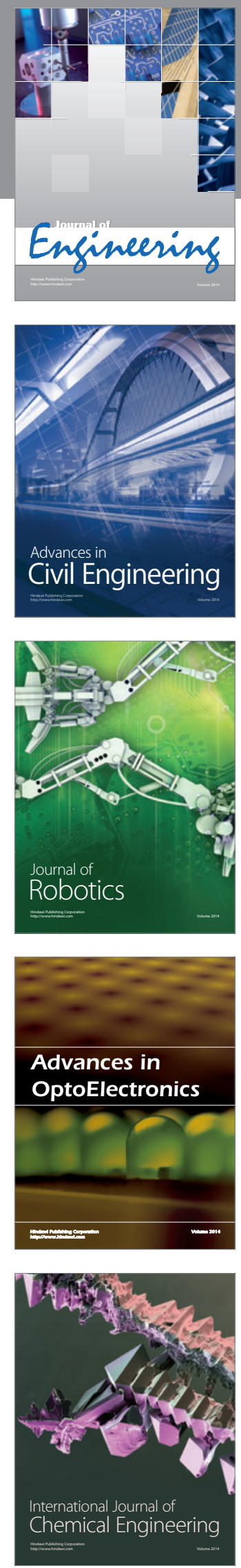

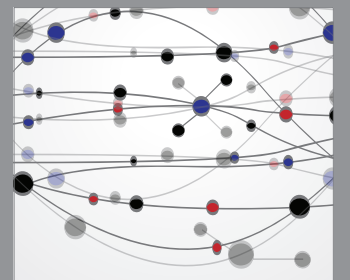

The Scientific World Journal
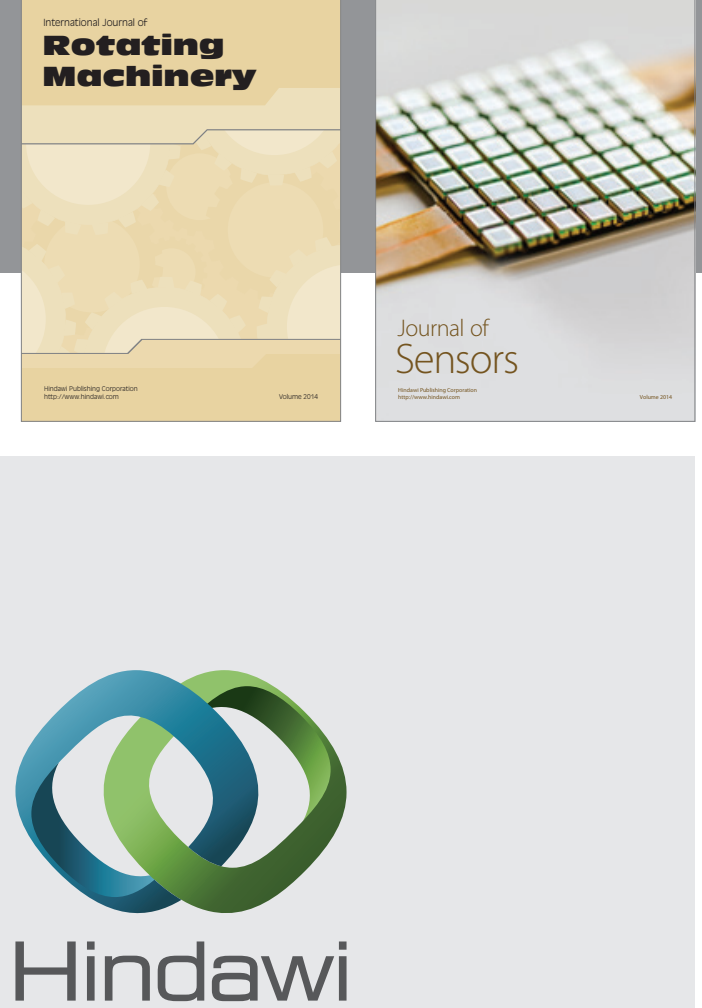

Submit your manuscripts at http://www.hindawi.com
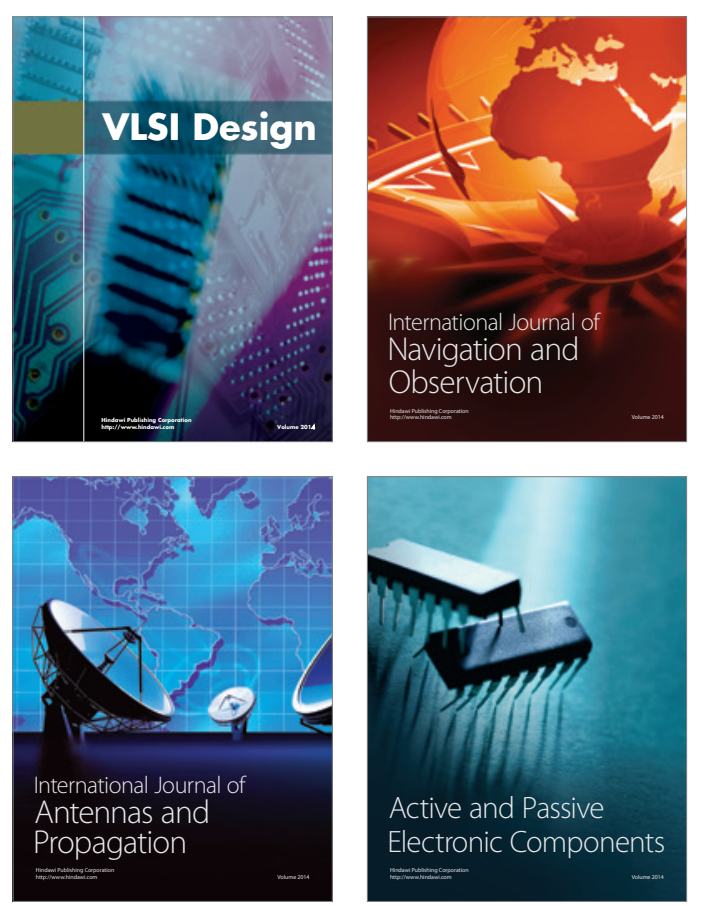
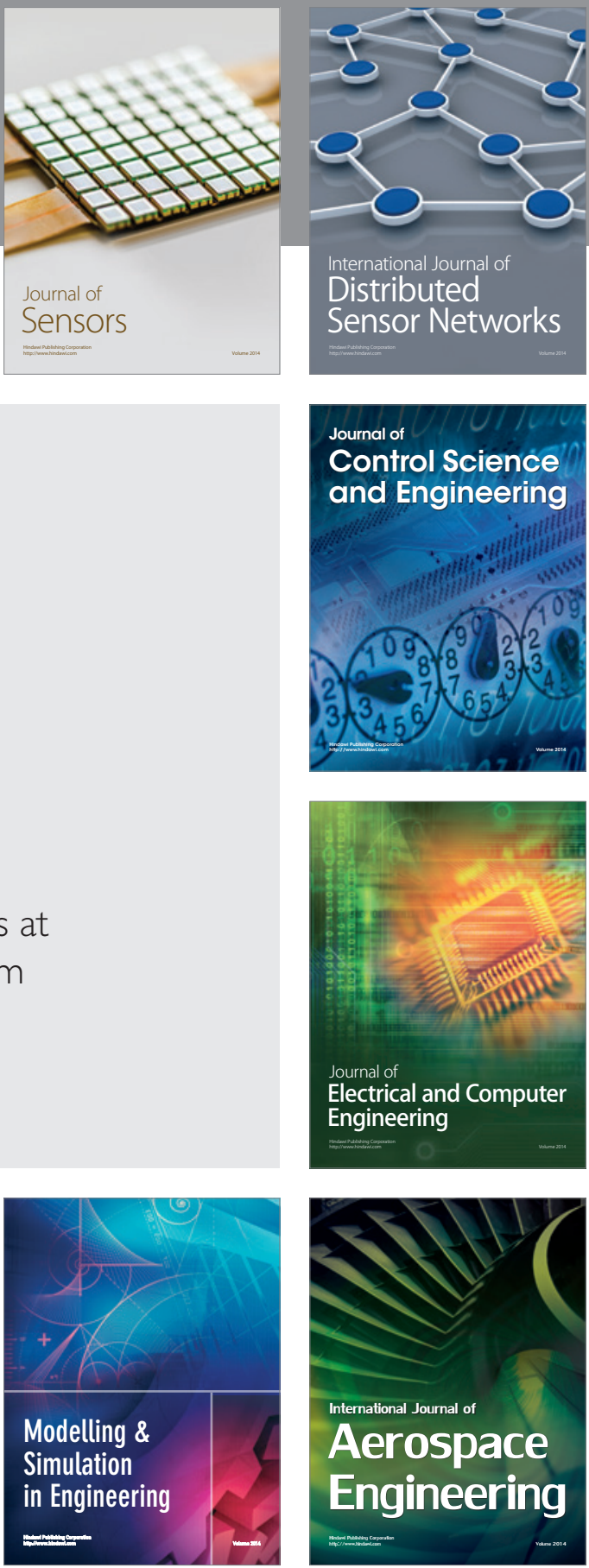

Journal of

Control Science

and Engineering
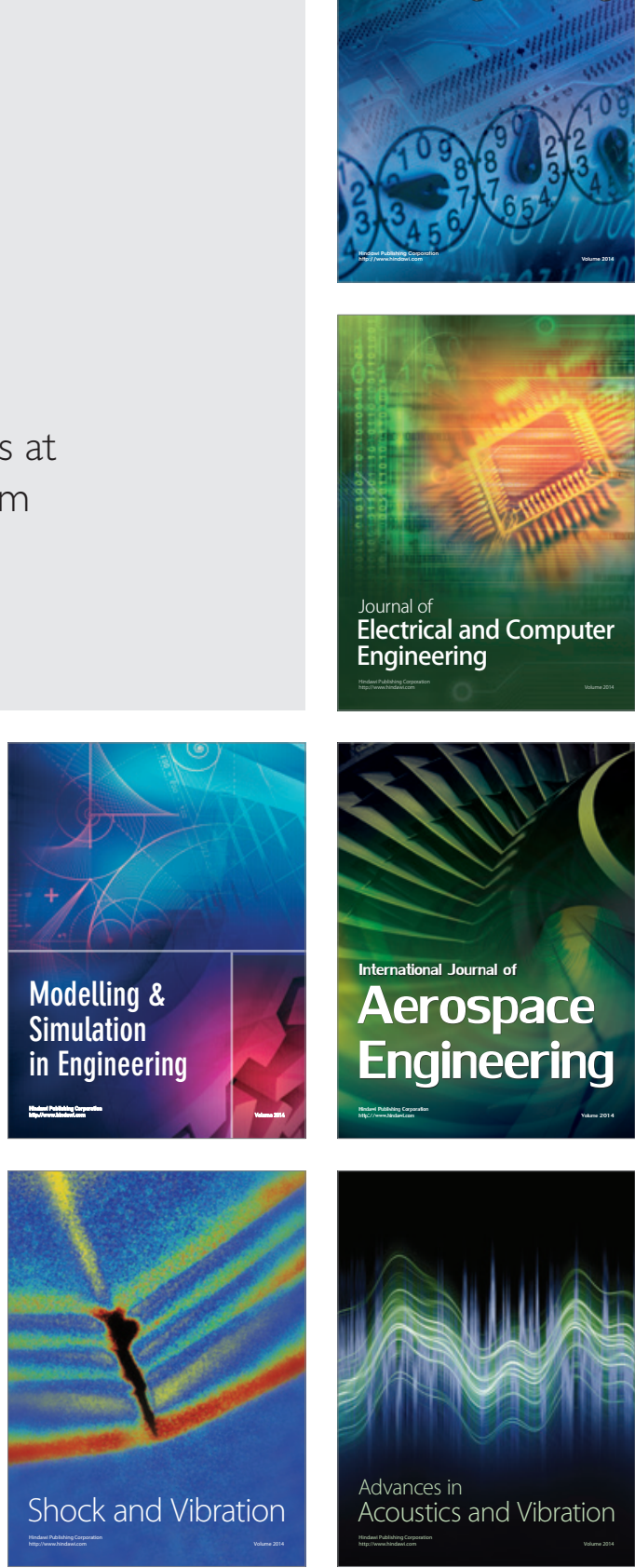